\title{
Ocular Manifestations in Colombian Patients with Systemic Rheumatologic Diseases
}

\author{
Pilar Uribe-Reina (D) ${ }^{1,2}$ \\ Juliana Muñoz-Ortiz $\mathbb{D}^{1,2}$ \\ Carlos Cifuentes-González' \\ Juliana Reyes-Guanes (D) ${ }^{2}$ \\ Juan Pablo Terreros- \\ Dorado (iD) \\ William Zambrano- \\ Romero (iD) ${ }^{1,3}$ \\ Carolina López-Rojas ${ }^{2}$ \\ Fabien Mantilla-Sylvain ${ }^{3}$ \\ Rubén Darío Mantilla- \\ Hernández ${ }^{3}$ \\ Alejandra de-la-Torre (iD) \\ 'Neuroscience Research Group \\ "NeURos", Escuela de Medicina \\ y Ciencias de la Salud, Universidad del \\ Rosario, Bogotá, Colombia; ${ }^{2}$ Escuela \\ Barraquer, Research Group, Escuela \\ Superior de Oftalmología del Instituto \\ Barraquer de América, Bogotá, \\ Colombia; ${ }^{3}$ Fundación Para la \\ Investigación en Dermatología \\ y Reumatología (FUNINDERMA), \\ Bogotá, Colombia
}

Purpose: To establish the prevalence of ocular involvement in a Colombian population with rheumatologic diseases.

Design: Observational cross-sectional study.

Methods: We included a probabilistic sample size of 797 patients who attended a rheumatologic disease center in Bogotá, Colombia. Statistical analysis with descriptive measures and Chi-square independence test between rheumatologic diseases and ophthalmological symptoms and diseases was performed.

Results: Eighty-four percent of the population were women, and the mean age was $54.61 \pm$ 15.64 years. The most common condition was rheumatoid arthritis $(33.37 \%)$, followed by fibromyalgia (22.71\%), Sjögren Syndrome (19.72\%), and systemic lupus erythematosus (9.91\%). Almost $7 \%$ of the patients presented polyautoimmunity. Thirty-five percent of the patients reported one or more ophthalmological symptoms, being dry eye sensation the most common $(30.86 \%)$, followed by ocular pain $(2.76 \%)$, red-eye, and decreased visual acuity (both $2.63 \%$ ). Similarly, $21.45 \%$ of the patients presented one or more ophthalmological diagnoses, being keratoconjunctivitis sicca the most common (15.93\%), followed by cataract, uveitis (1.38\% each), and scleritis (1.25\%).

Conclusion: Almost a third of the patients reported any ocular involvement. It is crucial to be aware of the most common ophthalmic manifestations among the different rheumatologic diseases in our population, to offer early specialist referral and timely treatment.

Keywords: rheumatology, ophthalmology, ophthalmic findings, keratoconjunctivitis sicca, prevalence

\section{Introduction}

Autoimmune diseases (ADs) are a heterogeneous group of disorders that can affect specific target organs or even organ systems due to the loss of tolerance to selfantigens. ${ }^{1}$ Many of these diseases share clinical signs, symptoms, physiopathological mechanisms, and genetic factors. ${ }^{2}$ The prevalence of ADs varies from study to study. Some studies calculate that ADs affects $5 \%$ of the American population, ${ }^{3}$ especially the female gender. They can compromise several systems such as the musculoskeletal, cardiovascular, pulmonary, hematopoietic, gastrointestinal, endocrine, central nervous system, and eyes. ${ }^{4,5}$

Within the extra-articular manifestations of rheumatologic diseases, different ocular pathologies have been described. These types of disease may affect the eye in different presentations during the natural course of the pathology, and frequently involve the eye as the first manifestation. ${ }^{6,7}$ An example can be rheumatoid arthritis (RA), which may debut with episcleritis. ${ }^{6}$ On the other hand, eye manifestations
Correspondence: Alejandra de-la-Torre Neuroscience Research Group - NeURos, Escuela de Medicina y Ciencias de la Salud, Universidad del Rosario, Carrera 24 \#

63C - 69, Bogotá, Colombia

Tel +573102482196

Email alejadelatorre@yahoo.com 
can reflect the inflammatory activity of an entity. For example, in inflammatory bowel disease, an episode of episcleritis is directly related to the pathology control. ${ }^{8}$ In addition, the eye is a sensitive indicator for potentially lethal occult systemic vasculitis in patients with RA who develop peripheral ulcerative keratitis or necrotizing scleritis. $^{9}$

Many ADs have been related to specific ocular manifestations, such as systemic lupus erythematosus (SLE), Sjögren syndrome (SS), Spondyloarthropathies, and Vasculitis associated with ANCAS, among others. ${ }^{10,11}$ The main structures affected in the eyes by systemic inflammatory diseases are the cornea, sclera, uvea, and retina, thus compromising vision. ${ }^{4,6}$

There is a significant impact on the life quality of patients with ADs. Besides, ocular manifestations in these patients can lead to important morbidity and worsen life quality, due to its symptoms and consequences. They can cause visual impairment and even blindness, ${ }^{12,13}$ so it is important to establish its epidemiology and characteristics to give them the attention they need. ${ }^{14}$

The present study aims to estimate the prevalence of ophthalmological compromise and the different clinical presentations in a group of patients with ADs from a rheumatology health center in Bogotá, Colombia.

\section{Methods}

\section{Design}

We conducted an observational descriptive cross-sectional study in patients who attended a rheumatologic disease center in Bogotá, Colombia, to document the presence of ophthalmic diseases and manifestations, from 2013 to 2019.

\section{Study Population}

Patients older than 18 years old, who attended the center were included. The exclusion criteria consisted of patients with ophthalmologic conditions not related to rheumatologic diseases.

To estimate the true proportion of adult patients with ocular manifestations in rheumatological disease, a simple random sampling for finite populations was used. Taking as reference what is reported in the literature, an expected proportion of $27 \%$, a population of 13.763 patients treated at the rheumatologic center, a confidence interval of $95 \%$ (taking a normal distribution critical point of 1.96), and an estimation error of 3\%, a sample size of 793 medical records was obtained. Based on the information available,
4 additional cases were considered for a total of 797 medical records. The sample size calculation was done using R Software 4.0.4 samplingbook-package ${ }^{15}$ (https:// www.R-for the estimation project.org/). All patients' diagnoses included in this study were classified according to the International Classification of Disease, tenth edition (ICD-10).

\section{Data Collection}

The evaluation and extraction of the medical records were performed by our trained personnel, for 4 months. We elaborated and validated a database in Microsoft Excel Microsoft (Microsoft Corp., Redmond, WA, USA) to record the information. Variables included were demographic, rheumatologic diseases, ocular symptoms, and ocular diagnosis.

\section{Statistical Analysis}

Following the data registration, the database was submitted for statistical analysis. The results were reported as means and standard deviation for continuous variables and frequency distribution tables for categorical variables. Associations between categorical variables were assessed using the Chi-square independence test between rheumatologic diseases and ophthalmological symptoms and diseases, with $95 \%$ and $99 \%$ confidence levels. All analyses were done in software $\mathrm{R}$ version 4.0.4.

\section{Bias Control}

Confounding bias may be considered because it is possible that the ocular diseases found during our review, were not necessarily related to rheumatologic diseases. Control of this type of bias was achieved through the design of a temporality variable, which indicated the appearance of ocular diseases concerning rheumatologic disease diagnosis. Although it is well known that some ADs may debut with ophthalmologic manifestations even before systemic affection, patients with ocular manifestations before ADs diagnosis were excluded.

\section{Results}

Most of the patients were female $(84.06 \%)$, and the mean age was 55 years. Most of the population was between 40 and 65 years of age. Analyzing some of the risk factors associated with autoimmune disease, family history of autoimmune disease and history of smoking were the most frequent. The least frequent risk factors were active smoking, silicon prosthesis, and tattoos, with percentual fractions below 7\%. Sociodemographic data are shown in Table 1. 
Table I Sociodemographic Data

\begin{tabular}{|l|c|}
\hline Variable & Data n (\%) \\
\hline Female & $670(84.06 \%)$ \\
Age (mean, SD) & $54.61 \pm 15.64$ years \\
Active smokers & $49(6.14 \%)$ \\
Past smokers & $178(22.33 \%)$ \\
Tattoos & $16(2 \%)$ \\
Silicon prosthesis & $22(2.76 \%)$ \\
Family history of autoimmune disease & $211(26.47 \%)$ \\
Mean age of autoimmune disease diagnosis & Female: 45.88 years \\
& Male: 50.656 years \\
\hline
\end{tabular}

Abbreviation: SD, Standard deviation.

A total of 45 different rheumatologic pathologies were found in the 797 patients, and in them, 1112 rheumatological diagnoses were registered (taking into account that a patient may present more than one diagnosis). Figure $1 \mathrm{~A}$ shows that the most common diagnoses were RA, followed by fibromyalgia (FM), SS, and SLE. It is important to highlight that almost $7 \%$ of the patients presented polyautoimmunity. Only those pathologies with 10 or more cases are graphically

\section{A Rheumatologic diagnoses}

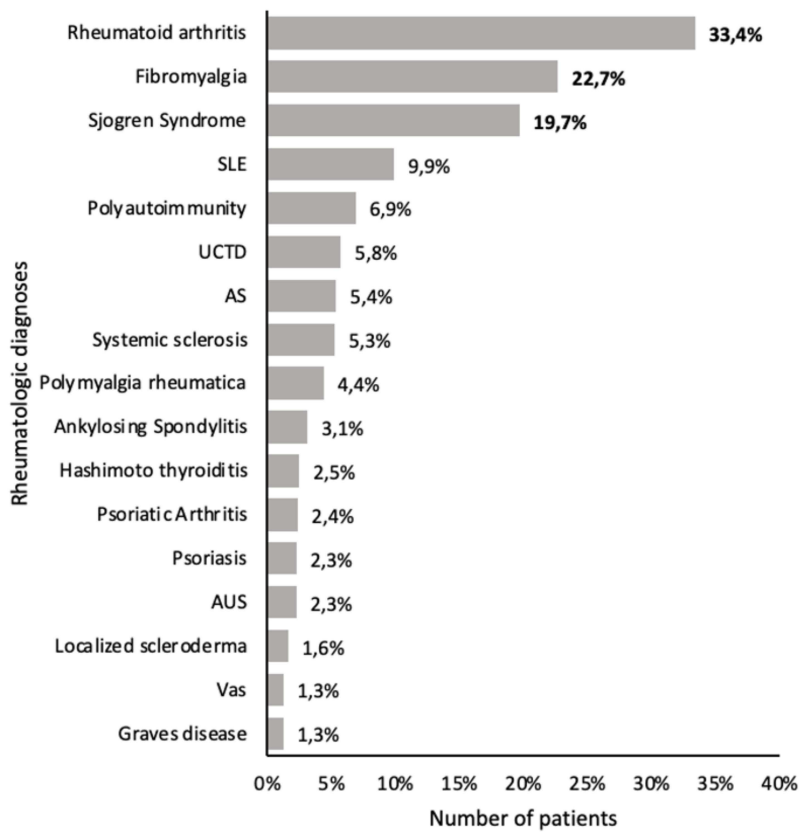

SLE: Systemic Lupus Erythematosus; UCTD: Undifferentiated connective tissue disease; AS: Antiphospholipid Syndrome; AUS: Axial undifferentiated spondylitis displayed. On the other hand, 21 different ophthalmological pathologies were found in the 797 patients, and 208 ophthalmological diagnoses were recorded in them. Figure 1B shows that the most common ophthalmological diagnosis was keratoconjunctivitis sicca. Those pathologies with less than 2 cases were graphically omitted.

All patients with Reiter syndrome (RS), Type 1 Diabetes, Large size vasculitis, Pernicious anemia, Celiac disease, Neuromyelitis optica (NMO), Pemphigoid, and Sclerosing cholangitis presented at least one ophthalmological symptom. Besides, almost $75 \%$ of patients with SS reported ophthalmological symptoms, followed by patients with Vasculitis associated with systemic disease (66.67\%), Autoimmune hepatitis (62.5\%), Multiple sclerosis (MS) (60\%), Hashimoto Thyroiditis (HT) (55\%), Myasthenia Gravis (50\%), polyautoimmunity (43.63\%) and SLE (43.03\%).

Regarding ophthalmological diagnosis, all the patients with celiac disease and NMO presented an ophthalmological diagnosis, followed by SS (78.34\%), vasculitis associated with systemic disease (66.67\%), small size

\section{B Ophthalmologic diagnoses}

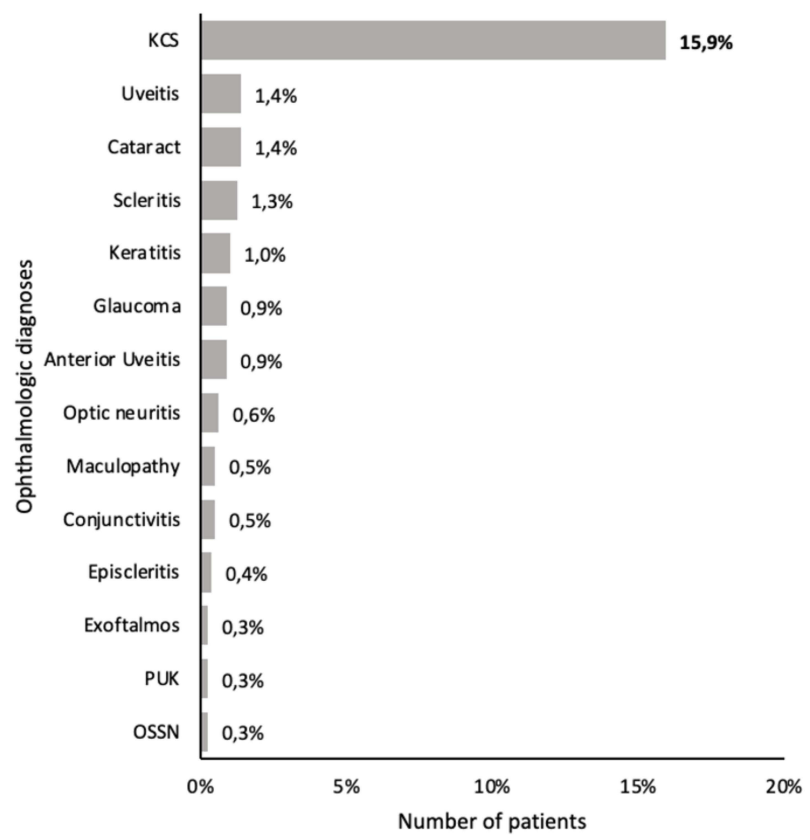

KCS: Keratoconjunctivitis sicca; PUK: Peripheral Ulcerative Keratitis

Figure I Most common rheumatologic and ophthalmologic diagnoses. 
vasculitis associated with ANCA (66.67\%), MS (60\%), Large size vessel vasculitis, Polymyositis (PM), and RS (50\% each). Additionally, $24.05 \%$ of the patients with SLE and $18.42 \%$ of the patients with RA had some form of ophthalmic manifestation.

It is important to mention that RA, SS, psoriatic arthritis, and psoriasis were statistically associated with the presence of ocular symptoms. In the same way, SS and polyautoimmunity had a statistically significant association with the presence of ophthalmological diagnosis. All rheumatologic diseases with their ophthalmological symptoms and diagnosis proportions are shown in Table 2.

From the total sample, $21.45 \%$ presented one or more ophthalmologic diagnoses, being KCS the most prevalent and the single common pathology among the 4 most prevalent rheumatologic diseases. KCS and keratitis were found to be statistically associated with SS. The ophthalmological diagnoses vs rheumatologic diseases data are shown in Table 3.

Thirty-five percent of the patients reported one or more ophthalmological symptoms, being dry eye (DE) sensation the most common (30.9\%), followed by ocular pain $(2.8 \%)$, red-eye $(2.6 \%)$, and decreased visual acuity (VA) (2.6\%). Considering the 4 most prevalent rheumatologic pathologies, we observe how dry eye, ocular pain, red eye, decreased VA, and photophobia were common in patients with these diagnoses. In particular, dry eye had a statistically significant association with SS, photophobia with SLE, and pruritus with FM. The ophthalmological symptoms vs rheumatologic diseases data are shown in Table 4.

An extension of Tables 3 and 4 are presented in Appendix 1 and Appendix 2.1 and 2.2, which show specific ocular symptoms and ophthalmologic diagnoses for each rheumatologic disease. Rheumatologic diseases with no ocular symptoms and/or ophthalmologic diagnoses were omitted.

\section{Discussion}

To the best of our knowledge, we present the fourth crosssectional study addressing ocular manifestations in rheumatologic diseases worldwide ${ }^{16-18}$ and the first in Latin America. We report an extended number of rheumatologic diseases, without excluding any reported previously.

As is well known, women tend to be more likely to have ADs. Even though we included both sex population, the female prevalence was $84.06 \%$. This agrees with the results of similar studies, which have reported a female prevalence of 71.2 to $91.96 \% .^{17,19}$

The mean age of our population was $54.61 \pm 15.64$ years. Similarly, the mean ages of $44.3 \pm 13.7$ and $48.9 \pm$ 19.3 have been reported in comparable studies. ${ }^{19,20}$ Contrarily, one study reported a mean age of $67.6 \pm 14.5$. $^{16}$

The most prevalent rheumatologic diseases found in our study were RA, FM, SS, and SLE. Similarly, data reported by Levitt et al coincides that RA is the most prevalent AD. ${ }^{16}$ Remarkably, polyautoimmunity represented $6.9 \%$ of our studied population. To the best of our knowledge, there is no available data to compare this result.

Our study reported one or more ophthalmological diagnoses in $21.45 \%$ of the population. Interestingly, $35 \%$ of the population reported at least one ophthalmologic symptom. Similar studies have reported ophthalmological manifestations in $2-7.7 \%$ of their studied population. ${ }^{16,17,20}$ Recently, a systematic review with meta-analysis by Turk et $\mathrm{al}^{21}$ corroborates our data, as it shows that the ocular involvement in ADs is between $20-33 \%$. These differences may be attributed to the inclusion of a greater number of rheumatologic diseases and ophthalmological diagnoses and symptoms compared to the mentioned similar studies. In the same way, the difference between the proportion of patients with ophthalmological symptoms and ophthalmological diagnosis could be attributed to ophthalmological disease sub-diagnosis.

In terms of ocular symptoms, we observed that the most frequent was DE in $30.86 \%$ of the patients. Similarly, Ausayakhun et $\mathrm{al}^{18}$ reported DE as the most prevalent manifestation, in $19.9 \%$ of the studied population.

Regarding ocular diagnosis, the most frequent was KCS (15.93\%), followed by cataract and uveitis. Contrarily, Ciurtin et $\mathrm{al}^{17}$ and Levitt et al ${ }^{16}$ reported anterior uveitis as the most frequent manifestation associated with HLA-B27 and Sarcoidosis, respectively. Our study reported a uveitis prevalence of $1.38 \%$, anterior uveitis prevalence of $0.88 \%$, posterior uveitis prevalence of $0.13 \%$, and panuveitis of $0.13 \%$.

Interestingly, we found that $6.9 \%$ of our population presented polyautoimmunity, which is defined as the concurrence of two ADs in the same patient. ${ }^{2}$ From them, almost $44 \%$ reported ocular symptoms, being DE the most common, and $42 \%$ presented an ophthalmological diagnosis. KCS was the most common diagnosis, found in $33.36 \%$ of the sample. Information is scarce regarding 
Table 2 Ocular Symptoms and Ophthalmological Diagnosis for Each Rheumatologic Disease

\begin{tabular}{|c|c|c|c|c|}
\hline & & Sample n (\%) & Ocular Symptoms & Ophthalmological Diagnosis \\
\hline \multicolumn{5}{|c|}{ Connective tissue disease } \\
\hline \multicolumn{2}{|l|}{ Rheumatoid arthritis } & $266(33.37 \%)$ & $80(30.08 \%) *$ & 49 (I8.42\%) \\
\hline \multicolumn{2}{|l|}{ Sjögren syndrome } & 157 (19.72\%) & $117(74.72 \%) * *$ & $123(78.34 \%) * *$ \\
\hline \multicolumn{2}{|c|}{ Systemic Lupus Erythematosus } & 79 (9.91\%) & $34(43.03 \%)$ & 19 (24.05\%) \\
\hline \multicolumn{2}{|l|}{ Polyautoimmunity } & $55(6.9 \%)$ & $24(43.63 \%)$ & $23(41.82 \%) * *$ \\
\hline \multicolumn{2}{|l|}{ Systemic sclerosis } & $42(5.27 \%)$ & $19(45.23 \%)$ & $9(21.43 \%)$ \\
\hline \multicolumn{2}{|l|}{ Localized Scleroderma } & $13(1.63 \%)$ & $3(23 \%)$ & I (7.7\%) \\
\hline \multicolumn{2}{|c|}{ Undifferentiated Connective Tissue Disease } & $46(5.78 \%)$ & $17(37 \%)$ & $6(13 \%)$ \\
\hline \multicolumn{2}{|c|}{ Juvenile Idiopathic Arthritis } & $5(0.62 \%)$ & 0 & 0 \\
\hline \multicolumn{2}{|c|}{ Mixed Connective Tissue Disease } & $4(0.5 \%)$ & I (25\%) & 0 \\
\hline \multicolumn{2}{|l|}{ Sarcoidosis } & $3(0.38 \%)$ & I (33.33\%) & 0 \\
\hline \multicolumn{5}{|l|}{ Spondyloarthropathies } \\
\hline \multicolumn{2}{|l|}{ Ankylosing Spondylitis } & $25(3.14 \%)$ & $4(16 \%)$ & $3(12 \%)$ \\
\hline \multicolumn{2}{|l|}{ Psoriatic Arthritis } & $19(2.38 \%)$ & I $(5.26 \%) *$ & $\mathrm{I}(5.2 \%)$ \\
\hline \multicolumn{2}{|c|}{ Axial undifferentiated spondylitis } & $18(2.26 \%)$ & $5(27.78 \%)$ & $3(16.66 \%)$ \\
\hline \multicolumn{2}{|c|}{ Undifferentiated peripheral spondylitis } & $5(0.63 \%)$ & 0 & 0 \\
\hline \multicolumn{2}{|c|}{ Reactive Arthritis } & $5(0.63 \%)$ & I (20\%) & 0 \\
\hline \multicolumn{2}{|l|}{ Reiter syndrome } & $2(0.25 \%)$ & $2(100 \%)$ & I (50\%) \\
\hline \multirow[t]{3}{*}{ Enteropathic arthritis } & Chron Disease & $3(0.38 \%)$ & I (33.3\%) & 0 \\
\hline & Ulcerative Colitis & I (0.125\%) & 0 & 0 \\
\hline & Celiac disease & I (0.125\%) & I (100\%) & I (I00\%) \\
\hline \multicolumn{5}{|l|}{ Myositis } \\
\hline \multicolumn{2}{|l|}{ Polymyalgia rheumatica } & $35(4.39 \%)$ & $10(28.57 \%)$ & 5 (14.29\%) \\
\hline \multicolumn{2}{|l|}{ Dermatomyositis } & 7 (0.88\%) & $3(42.85 \%)$ & $3(42.86 \%)$ \\
\hline \multicolumn{2}{|l|}{ Polymyositis } & $2(0.25 \%)$ & 0 & I (50\%) \\
\hline \multicolumn{5}{|c|}{ Autoimmune glandular disease } \\
\hline \multicolumn{2}{|l|}{ Hashimoto thyroiditis } & $20(2.5 \mathrm{I} \%)$ & II (55\%) & $6(30 \%)$ \\
\hline Graves' disease & & $10(1.25 \%)$ & I (I0\%) & $\mathrm{I}(10 \%)$ \\
\hline Type I Diabetes & & I (0.13\%) & I (100\%) & 0 \\
\hline Autoimmune hemato & liseases & & & \\
\hline Antiphospholipid Synd & & $43(5.4 \%)$ & $14(32.55 \%)$ & $8(18.6 \%)$ \\
\hline Autoimmune thrombo & nic purpura & $4(0.50 \%)$ & 0 & 0 \\
\hline Pernicious anemia & & $2(0.25 \%)$ & $2(100 \%)$ & 0 \\
\hline Autoimmune hemolyti & & I (0.13\%) & 0 & 0 \\
\hline Vasculitis & & & & \\
\hline Vasculitis associated $\mathrm{w}$ & bable etiology & $10(1.25 \%)$ & $3(30 \%)$ & $2(20 \%)$ \\
\hline Vasculitis associated $\mathrm{w}$ & stemic disease & $3(0.38 \%)$ & $2(66.67 \%)$ & $2(66.67 \%)$ \\
\hline Medium size vessel vas & & $3(0.38 \%)$ & I (33.33\%) & I (33.33\%) \\
\hline Small size vessel vascu & ociated with ANCA & $3(0.38 \%)$ & I (33.33\%) & $2(66.67 \%)$ \\
\hline Behcet Disease & & $3(0.38 \%)$ & I (33.33\%) & I (33.33\%) \\
\hline Large size vessel vascu & & $2(0.25 \%)$ & $2(100 \%)$ & I (50\%) \\
\hline Henoch-Schönlein pur & & I (0.13\%) & 0 & 0 \\
\hline
\end{tabular}


Table 2 (Continued).

\begin{tabular}{|c|c|c|c|}
\hline & Sample n (\%) & Ocular Symptoms & Ophthalmological Diagnosis \\
\hline \multicolumn{4}{|c|}{ Autoimmune dermatologic diseases } \\
\hline Psoriasis & $18(2.26 \%)$ & I $(5.55 \%) * *$ & $2(11.1 \%)$ \\
\hline Vitiligo & $3(0.38 \%)$ & I (33.3\%) & I (33.3\%) \\
\hline Pemphigoid & I (0.13\%) & I (100\%) & 0 \\
\hline \multicolumn{4}{|l|}{ Autoimmune liver diseases } \\
\hline Primary biliary cholangitis & $\mathrm{I}(0.25 \%)$ & 0 & I (50\%) \\
\hline Autoimmune hepatitis & $8(1 \%)$ & $5(62.5 \%)$ & $2(25 \%)$ \\
\hline Sclerosing cholangitis & I $(0.13 \%)$ & I (100\%) & 0 \\
\hline \multicolumn{4}{|c|}{ Autoimmune neurological diseases } \\
\hline Multiple Sclerosis & $5(0.63 \%)$ & $3(60 \%)$ & $3(60 \%)$ \\
\hline Neuromyelitis Optica & $2(0.25 \%)$ & $2(100 \%)$ & $2(100 \%)$ \\
\hline Myasthenia gravis & $2(0.25 \%)$ & I (50\%) & 0 \\
\hline \multicolumn{4}{|c|}{ Other rheumatologic diseases } \\
\hline Fibromyalgia & $18 \mid(22.7 \mid \%)$ & $63(34.8 \%)$ & $36(19.88 \%)$ \\
\hline
\end{tabular}

Notes: p-value is based in Chi-square proof with a $95 \%(*)$ and $99 \%(* *)$ confidence level.

Table 3 Ophthalmological Diagnoses Associated to the Main Rheumatologic Diseases

\begin{tabular}{|c|c|c|c|c|c|c|c|c|c|c|}
\hline \multirow[t]{2}{*}{$\begin{array}{l}\text { Rheumatologicl } \\
\text { Ophthalmological Diagnoses }\end{array}$} & \multicolumn{2}{|c|}{$\begin{array}{c}\text { Rheumatoid } \\
\text { Arthritis }\end{array}$} & \multicolumn{2}{|c|}{ Sjögren Syndrome } & \multicolumn{2}{|c|}{ SLE } & \multicolumn{2}{|c|}{ Fibromyalgia } & \multicolumn{2}{|c|}{ Total Per Symptom } \\
\hline & Ocurrence & p-value & Ocurrence & p-value & Ocurrence & p-value & Ocurrence & p-value & Ocurrence & $\%$ \\
\hline Keratoconjunctivitis sicca & 34 (12.78\%) & 0.1 & $12 \mid(77.07 \%)$ & $0.0001 * *$ & $16(20.25 \%)$ & 0.34 & $28(15.46 \%)$ & 0.93 & 127 & $15.93 \%$ \\
\hline Uveitis & $3(1.13 \%)$ & 0.91 & $2(1.27 \%)$ & 1 & 0 & & I (0.55\%) & 0.93 & 11 & $1.38 \%$ \\
\hline Cataract & $4(1.5 \%)$ & I & I (0.63\%) & 0.6 & I (I.26\%) & & I (0.55\%) & 0.46 & 11 & $1.38 \%$ \\
\hline Scleritis & $6(2.25 \%)$ & 0.14 & $3(1.91 \%)$ & 0.67 & 0 & & 0 & 0.17 & 10 & $1.25 \%$ \\
\hline Keratitis & $2(0.75 \%)$ & 0.89 & $6(3.82 \%)$ & $0.0004 * *$ & $2(2.5 \%)$ & 0.4 & $2(1.1 \%)$ & 1 & 8 & $1.0 \%$ \\
\hline Glaucoma & $2(0.75 \%)$ & I & $2(1.27 \%)$ & 0.9 & 0 & 0.8 & $2(1.1 \%)$ & 1 & 7 & $0.88 \%$ \\
\hline Anterior uveitis & I (0.37\%) & 0.49 & $2(1.27 \%)$ & 0.9 & 0 & & 0 & & 7 & $0.88 \%$ \\
\hline Optic neuritis & 0 & & $2(1.27 \%)$ & 0.56 & $2(2.5 \%)$ & 0.13 & I (0.55\%) & 1 & 5 & $0.63 \%$ \\
\hline Maculopathy & $3(1.13 \%)$ & 0.21 & I (0.63\%) & 1 & 0 & & $2(1.1 \%)$ & 0.47 & 4 & $0.5 \%$ \\
\hline Conjunctivitis & I (0.37\%) & 1 & I (0.63\%) & I & I (1.26\%) & 0.86 & 0 & & 4 & $0.5 \%$ \\
\hline Episcleritis & I $(0.37 \%)$ & I & I $(0.63 \%)$ & 1 & 0 & & 0 & & 3 & $0.38 \%$ \\
\hline Exoftalmos & 0 & & 0 & & 0 & & 0 & & 2 & $0.25 \%$ \\
\hline Peripheral ulcerative keratitis & I (0.37\%) & I & I (0.63\%) & 0.85 & 0 & & 0 & & 2 & $0.25 \%$ \\
\hline Ocular Surface Squamous Neoplasia & $2(0.75 \%)$ & 0.21 & 0 & & 0 & & 0 & & 2 & $0.25 \%$ \\
\hline Retinal vasculitis & 0 & & 0 & & 0 & & 0 & & 1 & $0.13 \%$ \\
\hline Panuveitis & 0 & & 0 & & 0 & & 0 & & 1 & $0.13 \%$ \\
\hline Posterior uveitis & 0 & & 0 & & 0 & & 0 & & 1 & $0.13 \%$ \\
\hline Corneal perforation & I (0.37\%) & 0.72 & I (0.63\%) & 0.44 & 0 & & 0 & & 1 & $0.13 \%$ \\
\hline Macular edema & I (0.37\%) & 0.72 & 0 & & 0 & & 0 & & I & $0.13 \%$ \\
\hline Central retinal artery ocussion & 0 & & I (0.63\%) & 0.44 & 0 & & I $(0.63 \%)$ & 0.44 & 1 & $0.13 \%$ \\
\hline Blind eye & 0 & & 0 & & 0 & & 0 & & 1 & $0.13 \%$ \\
\hline Retinal detachment & I (0.37\%) & 0.72 & I (0.63\%) & 0.44 & 0 & & I (0.63\%) & 0.44 & 1 & $0.13 \%$ \\
\hline
\end{tabular}

Notes: ${ }^{*}$ p-value is based in chi squared proof with a $99 \%$ confidence level. 
Table 4 Ophthalmological Symptoms Associated to the Main Rheumatologic Diseases

\begin{tabular}{|c|c|c|c|c|c|c|c|c|c|c|}
\hline \multirow[t]{2}{*}{ Disease/Symptom } & \multicolumn{2}{|c|}{ Rheumatoid Arthritis } & \multicolumn{2}{|c|}{ Sjögren Syndrome } & \multicolumn{2}{|c|}{ SLE } & \multicolumn{2}{|c|}{ Fibromyalgia } & \multicolumn{2}{|c|}{ Total Per Symptom } \\
\hline & Ocurrence & p-value & Ocurrence & p-value & Ocurrence & p-value & Ocurrence & p-value & Ocurrence & $\%$ \\
\hline Dry eye & $72(27 \%)$ & 0.106 & 113 (72.43\%) & $0.000 * *$ & 28 (35.9\%) & 0.385 & 57 (31.49\%) & 0.928 & 246 & $30.86 \%$ \\
\hline Ocular pain & 9 (3.4\%) & 0.595 & $4(2.56 \%)$ & 1,00 & $\mathrm{I}(\mathrm{I} .27 \%)$ & 0.616 & $3(1.67 \%)$ & 0.446 & 22 & $2.76 \%$ \\
\hline Red eye & $10(3.77 \%)$ & 0.244 & $6(3.82 \%)$ & 0.456 & $2(2.5 \%)$ & 1,00 & $3(1.66 \%)$ & 0.497 & 21 & $2.63 \%$ \\
\hline Decreased VA & $4(1.5 \%)$ & 0.236 & $6(3.82 \%)$ & 0.452 & $2(2.5 \%)$ & 1,00 & $2(1.10 \%)$ & 0.229 & 21 & $2.63 \%$ \\
\hline Photophobia & $2(0.75 \%)$ & 0.213 & 3 (1.91\%) & 1,00 & $6(7.6 \%)$ & $0.000 * *$ & I (0.55\%) & 0.276 & 14 & $1.76 \%$ \\
\hline Floaters & $3(1.13 \%)$ & 1,00 & I (0.63\%) & 0.815 & 0 & & $3(1.66 \%)$ & 0.716 & 9 & $1.13 \%$ \\
\hline Burning & $3(1.12 \%)$ & 0.668 & $2(1.27 \%)$ & 0.745 & 0 & & & & 6 & $0.75 \%$ \\
\hline Foreign body sensation & I (0.37\%) & 0.872 & $2(1.27 \%)$ & 0.564 & 0 & & 0 & & 5 & $0.63 \%$ \\
\hline Tearing & $3(1.12 \%)$ & 0.431 & $2(1.27 \%)$ & 0.563 & 0 & & $2(1.10 \%)$ & 0.697 & 5 & $0.63 \%$ \\
\hline Pruritus & $2(0.75 \%)$ & 0.863 & I (0.63\%) & 1,00 & 0 & & $3(1.66 \%)$ & $0.057^{*}$ & 4 & $0.5 \%$ \\
\hline Diplopia & 0 & & 0 & & I (I.27\%) & 0.475 & 0 & & 2 & $0.25 \%$ \\
\hline
\end{tabular}

Notes: ${ }^{* *}$ p-value is based in chi squared proof with a $95 \%$ confidence level. * p-value asymptotically significant for a $95 \%$ confidence level.

ocular manifestations in polyautoimmunity. We hypothesize that this could be attributed to genetic and epigenetic factors. To the best of our knowledge, we present the first ocular involvement prevalence for this condition.

\section{Ocular Manifestations in the Most Prevalent Diseases}

RA was the most common diagnosis in our sample, with 266 (33.37\%) patients. Regarding ocular symptoms, $30.08 \%$ reported at least one, being DE the most common (27\%), followed by red eye, ocular pain, decreased VA, floaters, burning, and tearing. The most common diagnoses were KCS (12.78\%) and scleritis (2.25\%), followed by cataract, uveitis, and maculopathy. Among extraarticular RA manifestations, ophthalmologic involvement is often significant and causes diverse degrees of ocular morbidity. Its prevalence varies between studies, ranging from $10-58 \% .{ }^{22-25}$ A systematic review and meta-analysis by Turk et $\mathrm{al}^{21}$ reported a prevalence of $18 \%$. DE and KCS have been reported as the most common ocular manifestation (10-58\%), followed by scleritis $(1-5 \%),{ }^{21-30}$ which is very similar to our results. Other associated ocular manifestations are anterior uveitis (4\%), ${ }^{31}$ keratitis $(3 \%),{ }^{23}$ and PUK $(<1 \%){ }^{24}$

SS was found in $19.72 \%$ of our sample. Almost $75 \%$ presented at least one ocular symptom and nearly $79 \%$ had an ophthalmologic diagnosis. The most common symptom was DE, followed by red-eye, decreased VA, and ocular pain. The most common diagnosis was KCS (77.07\%), followed by keratitis, and scleritis. Ocular compromise in SS is well described in the literature. The characteristic phenotype of SS includes ocular and oral dryness due to autoimmune inflammation of lacrimal and salivary glands. ${ }^{32}$ The prevalence of ocular involvement in SS was reported to be $89 \%$ in a recent systematic review, ${ }^{21}$ which is slightly higher than the one we found. Other studies report even higher prevalence, ranging from 93-96\%. ${ }^{33,34}$ As expected, the most common diagnosis was KCS, as it has been reported. ${ }^{32,35-37}$ Similarly, other ophthalmologic diagnoses had been described. Akpek et al reported the following ocular involvement in a group of SS patients: corneal perforation (3.1\%), corneal ulcer $(0.6 \%)$, corneal scarring $(4.3 \%)$, papillary conjunctivitis $(7.4 \%)$, follicular conjunctivitis $(2.5 \%)$, uveitis $(1.2 \%)$, scleritis/episcleritis $(0.6 \%)$, optic neuropathy $(1.8 \%)$, and orbital inflammation $(1.8 \%){ }^{14}$

SLE diagnosis was found in 79 patients $(9.91 \%)$ of our sample. Thirty-four patients reported ocular symptoms, being $\mathrm{DE}$ the most common, followed by photophobia, red-eye, and decreased VA. Similarly, $20.25 \%$ of the patients had KCS diagnosis, $2.5 \%$ keratitis and $\mathrm{ON}$, and $1.26 \%$ conjunctivitis and cataract. It is well known that ocular manifestations can be found in nearly one-third of SLE patients, ${ }^{21,38-42}$ which is similar to our results. SLE can affect any part of the eye and its severity can range from mild manifestations to severe, being a sightthreatening disease. ${ }^{39}$ As our results, it is reported that the most common ocular manifestation in SLE is $\mathrm{KCS},{ }^{41,43}$ which can affect from one-quarter to one-third of SLE patients. ${ }^{44}$ Another common ocular manifestation is retinal vasculitis; in a study by Stafford-Brady et al, 7\% of patients with SLE developed retinal vasculitis. ${ }^{45}$ Interestingly, none of our patients had this diagnosis. On the other hand, neuro-ophthalmic manifestations of lupus 
Table 5 Literature Reported Ophthalmological Symptoms and Diseases Associated to Rheumatologic Diseases

\begin{tabular}{|c|c|c|}
\hline Rheumatologic Disease & & Literature \\
\hline \multicolumn{3}{|l|}{ Connective tissue disease } \\
\hline Rheumatoid arthritis & \multicolumn{2}{|c|}{$\begin{array}{c}\text { Ocular involvement } 10-58 \%,{ }^{21-25} \text { Dry eye } 10-58 \%,{ }^{21-25} \text { Scleritis } 1-5 \%,{ }^{21-30} \text { Anterior uveitis } 4 \%,{ }^{31} \text { Keratitis } \\
3 \%,{ }^{23} \text { PUK }<1 \%{ }^{24}\end{array}$} \\
\hline Sjögren syndrome & \multicolumn{2}{|c|}{$\begin{array}{c}\text { Ocular involvement } 89-96 \%,{ }^{21,33,34} \text { Dry eye and KCS the most common } 90-97 \%,{ }^{21,32,34-37} \text { Corneal scarring } \\
\begin{array}{c}\text { 4.3\%, }{ }^{14} \text { Corneal perforation } 3.1 \%,{ }^{14} \text { ON } 1.8 \%,{ }^{14} \text { Uveitis } 1.2 \%,{ }^{14} \text { Corneal ulcer } 0.6 \%,{ }^{14} \text { Scleritis/episcleritis } \\
0.6 \%{ }^{14}\end{array}\end{array}$} \\
\hline Systemic Lupus Erythematosus & \multicolumn{2}{|c|}{$\begin{array}{l}\text { Ocular involvement in one third of the patients, }{ }^{21,38-42} \mathrm{KCS} \text { most common ocular manifestation } 25 \%,{ }^{41,43,44} \\
\text { Retinal Vasculitis 7\%, }{ }^{45} \text { ON I\%, }{ }^{45} \text { Uncommon, PUK, },{ }^{39} \text { Scleritis, }{ }^{39} \text { Epiescleritis, }{ }^{39} \text { Choroidopathy with serous } \\
\text { detachments, }{ }^{39} \text { Orbit myositis, }{ }^{39} \text { Internuclear Ophthalmoplegia, }{ }^{39} \text { Blepharospasm, }{ }^{39} \text { Retinal } \\
\text { Necrosis, }{ }^{39} \text { Uveitis }{ }^{40}\end{array}$} \\
\hline Polyautoimmunity & \multicolumn{2}{|c|}{$\begin{array}{l}\text { Dry eye Secondary Sjögren Syndrome and rheumatoid arthritis } 4-18 \%,{ }^{51,52} \text { Dry eye Secondary Sjögren } \\
\text { Syndrome and Systemic Lupus Erythematosus II\%, }{ }^{51} \text { Thyroid-associated orbitopathy Celiac disease and HT } \\
\text { or GD, }{ }^{53} \text { Dry eye Secondary Sjögren Syndrome and Primary Biliary Cirrhosis }{ }^{54}\end{array}$} \\
\hline Systemic sclerosis & \multicolumn{2}{|c|}{$\begin{array}{l}\text { Pinguecula } 82.2 \%,{ }^{55} \text { KCS } 14.2-79 \%,{ }^{17,56,57} \text { Eyelid stiffness } 29-65 \%,{ }^{57-59} \text { Astigmatism } 55 \%,{ }^{60} \text { Glaucoma } \\
23 \%,{ }^{55} \text { Loss of sackcloth } 15.6 \%,{ }^{55} \text { Uveitis } 3 \%{ }^{60}\end{array}$} \\
\hline Localized Scleroderma & \multicolumn{2}{|r|}{$\begin{array}{l}\text { Ocular manifestations } 2.1-6,7 \%,{ }^{61,62} \text { Glaucoma }{ }^{63} \text { Uveitis, }{ }^{61,62} \text { Eyelid/eyelash } \\
\text { involvement, }{ }^{62} \text { Epiescleritis, }{ }^{61,62} \text { Dry eye, }{ }^{61} \text { Keratitis, }{ }^{61} \text { Papiledema }{ }^{61}\end{array}$} \\
\hline $\begin{array}{l}\text { Undifferentiated Connective Tissue } \\
\text { Disease }\end{array}$ & \multicolumn{2}{|r|}{ Scleritis, ${ }^{64} \mathrm{ON},{ }^{65}$ Corneal thinning ${ }^{66}$} \\
\hline Juvenile Idiopathic Arthritis & \multicolumn{2}{|r|}{ Uveitis $17 \%-12 \%,{ }^{67,68}$ Band keratopathy $7-70 \%,{ }^{69,70}$ Ocular hypotony $19 \%,{ }^{70} \mathrm{KCS} 8.5 \%{ }^{70}$} \\
\hline Mixed Connective Tissue Disease & \multicolumn{2}{|r|}{ KCS (48\%), ${ }^{71}$ Maculopathy (5\%), ${ }^{72}$ Retinal vasculitis, ${ }^{73,74} \mathrm{NO}^{75}$} \\
\hline Sarcoidosis & \multicolumn{2}{|r|}{ Ocular involvement $13-79 \%,{ }^{21,76-78}$ Uveitis, $^{79}$ Scleritis, ${ }^{80}$ Dry eye, ${ }^{79,80}$ Conjunctival nodules ${ }^{79}$} \\
\hline \multicolumn{3}{|l|}{ Spondyloarthropathies } \\
\hline Ankylosing Spondylitis & & Uveitis $10-50 \%,{ }^{17,81-83}$ Dry eye ${ }^{84}$ \\
\hline Psoriatic Arthritis & \multicolumn{2}{|c|}{$\begin{array}{c}\text { Ocular involvement } 22.5-31.25 \%,{ }^{85,86} \text { KCS } 15 \%,{ }^{86} \text { Glaucoma } 10 \%,{ }^{86} \text { Cataract } 10 \%,{ }^{86} \text { Pinguecula } 20.0 \%,{ }^{86} \\
\text { Pterygium } 5 \%,{ }^{86} \text { Uveitis } 5 \%{ }^{85,86}\end{array}$} \\
\hline Axial undifferentiated spondylitis & \multicolumn{2}{|r|}{ Uveitis $17 \%,{ }^{87}$ Dry eye, ${ }^{84}$} \\
\hline $\begin{array}{l}\text { Undifferentiated peripheral } \\
\text { spondylitis }\end{array}$ & \multicolumn{2}{|r|}{ Uveitis $8 \%^{88}$} \\
\hline Reactive Arthritis & \multirow{2}{*}{\multicolumn{2}{|c|}{ Conjunctivitis $10.52 \%,{ }^{17}$ Uveitis $5.26 \%{ }^{17}$}} \\
\hline Reiter syndrome & & \\
\hline \multirow[t]{3}{*}{ Enteropathic arthritis } & $\begin{array}{l}\text { Chron } \\
\text { Disease }\end{array}$ & \multirow[t]{2}{*}{$\begin{array}{c}\text { Ocular involvement } 10-43 \%,{ }^{89,90} \text { Ocular inflammatory disorders } 0.3-13 \%,{ }^{8} \text { Episcleritis (2- } \\
5 \%),{ }^{8} \text { Uveitis(0.5\%-3.5\%), }{ }^{8} \text { Scleritis; }{ }^{8} \mathrm{KCS}^{8}\end{array}$} \\
\hline & $\begin{array}{l}\text { Ulcerative } \\
\text { Colitis }\end{array}$ & \\
\hline & $\begin{array}{l}\text { Celiac } \\
\text { disease }\end{array}$ & Dry eye, ${ }^{53}$ Cataract, ${ }^{53}$ Uveitis, ${ }^{53}$ NO, ${ }^{53}$ Orbital myositis, ${ }^{53}$ CRVO $^{53}$ nyctalopia $^{53}$ \\
\hline \multicolumn{3}{|l|}{ Myositis } \\
\hline Polymyalgia rheumatica & & Uveitis, $^{11,91}$ Scleritis and necrotizing scleritis, ${ }^{11,91,92}$ Episcleritis ${ }^{11,92,93}$ \\
\hline
\end{tabular}


Table 5 (Continued).

\begin{tabular}{|c|c|}
\hline Rheumatologic Disease & Literature \\
\hline Dermatomyositis & Dry eye, ${ }^{94}$ Macular edema, ${ }^{94}$ Retinitis, ${ }^{94}$ CRAO ${ }^{94}$ \\
\hline Polymyositis & Dry eye ${ }^{18}$ \\
\hline \multicolumn{2}{|l|}{ Autoimmune glandular disease } \\
\hline Hashimoto thyroiditis & Thyroid-associated ophthalmopathy $22 \%,{ }^{95-97}$ Dry eye, ${ }^{96,97}$ Proptosis, ${ }^{95-97}$ Ocular myopathy ${ }^{95-97}$ \\
\hline Graves' disease & Ocular involvement $67 \%,{ }^{98}$ Thyroid-associated ophthalmopathy $25 \%,{ }^{99}$ Dry eye $11 \%,{ }^{98}$ Proptosis, ${ }^{98}$ Lid lag $^{98}$ \\
\hline Type I Diabetes & Macular edema, ${ }^{100}$ Glaucoma, ${ }^{100}$ RD; ${ }^{100}$ Cataract, ${ }^{100}$ Dry eye ${ }^{101,102}$ \\
\hline \multicolumn{2}{|l|}{ Autoimmune hematologic diseases } \\
\hline Antiphospholipid Syndrome & $\begin{array}{c}\text { Ocular involvement 8-88\%, }{ }^{21,103-106} \mathrm{CRVO},{ }^{104,107-109} \mathrm{CRAO},{ }^{104,107-109} \text { Branch retinal vein occlusion, }{ }^{104,107-} \\
{ }^{109} \text { Branch retinal artery occlusion, }{ }^{104,107-109} \mathrm{KCS},{ }^{104} \text { Anterior uveitis, }{ }^{110} \text { Retinal vasculitis, }{ }^{110} \text { Scleritis and } \\
\text { necrotizing scleritis }{ }^{106,111}\end{array}$ \\
\hline $\begin{array}{l}\text { Autoimmune thrombocytopenic } \\
\text { purpura }\end{array}$ & $\begin{array}{c}\text { Spontaneous bilateral peripapillary, }{ }^{1 / 2} \text { subhyaloid and vitreous hemorrhage, }{ }^{1 / 2} \text { Massive subretinal } \\
\text { hemorrhage, }{ }^{1 / 3} \text { Purtscher's retinopathy; }{ }^{1 / 4} \text { Progressive retinopathy, }{ }^{1 / 5} \text { Valsalva retinopathy, }{ }^{116} \\
\text { Suprachoroidal hemorrhage simulating melanoma }\end{array}$ \\
\hline Pernicious anemia & ON, ${ }^{118,119}$ Delayed visual evoked potential, ${ }^{120}$ Retrobulbar neuritis ${ }^{121,122}$ \\
\hline Autoimmune hemolytic anemia & Retinal phlebitis, ${ }^{123}$ Bilateral macular hemorrhage ${ }^{124}$ \\
\hline \multicolumn{2}{|l|}{ Vasculitis } \\
\hline $\begin{array}{l}\text { Vasculitis associated with probable } \\
\text { etiology }\end{array}$ & No prevalence data \\
\hline $\begin{array}{l}\text { Vasculitis associated with a systemic } \\
\text { disease }\end{array}$ & Episcleritis, ${ }^{125}$ Scleritis, ${ }^{125}$ PUK, ${ }^{125}$ Uveitis, ${ }^{125}$ Retinopathy ${ }^{125}$ \\
\hline Medium size vessel vasculitis & $\begin{array}{c}\text { CRAO, }{ }^{126,127} \text { ON, }{ }^{126} \text { Scleritis, }{ }^{127} \text { Non granulomatous uveitis, }{ }^{127} \text { Retinal vasculitis, }{ }^{127} \text { PUK, }{ }^{127} \text { Pseudotumor } \\
\text { of the orbit }{ }^{127}\end{array}$ \\
\hline $\begin{array}{l}\text { Small size vessel vasculitis associated } \\
\text { with ANCA }\end{array}$ & $\begin{array}{c}\text { Ocular involvement } 12.5-15.62 \%,{ }^{16,128} \text { ON } 50 \%,{ }^{128} \text { Scleritis } 28 \%,{ }^{128} \text { Iritis } 28 \%,{ }^{128} \text { Retinal vasculitis } 17 \%,{ }^{128} \\
\text { Oculomotor disorder } 17 \%,{ }^{128} \text { PUK } 11 \%{ }^{128}\end{array}$ \\
\hline Behcet Disease & $\begin{array}{c}\text { Cataract } 30.3 \%,{ }^{129} \text { Panuveitis } 20 \%,{ }^{129} \text { Posterir uveitis } 15.1 \%,{ }^{129} \text { Anterior uveitis } 63.2 \%,{ }^{130} \text { Periphebitis } \\
65 \%,{ }^{130} \text { Optic atrophy, }{ }^{129} \text { RD, }{ }^{129} \text { Macular edemar, }{ }^{129} \text { Papillary edema, }{ }^{129} \text { Ocular hypertonia, }{ }^{129} \text { retinal } \\
\text { ischemia }{ }^{129}\end{array}$ \\
\hline Large size vessel vasculitis & $\begin{array}{l}\text { Anterior ischemic optic neuropathy } 81 \%,{ }^{131} \text { Ocular involvement } 40-80 \%,{ }^{132} \text { Amaurosis fugax } 25.6- \\
30.6 \%,{ }^{131,133} \text { Cilioretinal artery occlusion } 22 \%,{ }^{131} \text { CRAO } 14 \%,{ }^{131} \text { Posterior ischemic optic neuropathy } 7 \%{ }^{131}\end{array}$ \\
\hline Henoch-Schönlein purpura & $\begin{array}{c}\text { Anterior uveitis, }{ }^{134} \text { Anterior ischemic neuropthy, }{ }^{135} \text { Cystoid macular edema, }{ }^{136} \text { Retinal hemorrhages, }{ }^{136} \\
\text { Cotton wool spots, }{ }^{136} \text { Episcleritis }{ }^{137}\end{array}$ \\
\hline \multicolumn{2}{|c|}{ Autoimmune dermatologic diseases } \\
\hline Psoriasis & $\begin{array}{l}\text { Ocular involvement } 10-67 \%,{ }^{138-140} \text { Anterior uveitis } 82 \%,{ }^{85} \text { Bilateral uveitis } 64 \%,{ }^{85} \text { Cataract } 28-30.2 \%,{ }^{85,141} \\
\text { KCS } 16.28 \%,{ }^{141} \text { Posterior uveitis } 9.9 \%,{ }^{85} \text { Macular edema } 7.2 \%,{ }^{85} \text { Intermediate uveitis } 3.3 \%,{ }^{85} \text { Central serous } \\
\text { chorioretinopathy } 2.4 \%,{ }^{85} \text { Episcleritis } 2.4 \%,{ }^{85} \text { Glaucoma } 2.3 \%,{ }^{141} \text { Retinal vasculitis } 1.6 \%{ }^{85}\end{array}$ \\
\hline Vitiligo & $\begin{array}{l}\text { Ocular involvement } 22.2-66.6 \%,{ }^{142,143} \text { Pigmentation on anterior chamber } 18 \%,{ }^{143} \text { Retinal pigment } \\
\text { epithelium hypopigmentation } 9 \%,{ }^{43} \text { Uveitis } 5 \%,{ }^{143} \text { Hypopigmented spots on the iris } 3 \%,{ }^{143} \mathrm{KCS}^{143,144}\end{array}$ \\
\hline
\end{tabular}


Table 5 (Continued).

\begin{tabular}{|c|c|}
\hline Rheumatologic Disease & Literature \\
\hline Pemphigoid & $\begin{array}{l}\text { Conjunctival erosions } 33.3 \%,{ }^{145,146} \text { Epiphora } 23.9 \%,{ }^{145} \text { Eyelid and medial epicanthus erosion } 20.8- \\
41.6 \%,{ }^{145,146} \text { Dry eye; }{ }^{146} \text { Conjunctival fibrosis, }{ }^{145,146} \text { Symblepharon, }{ }^{146} \text { Ankyloblepharon, }{ }^{146} \text { Frozen eye }{ }^{146}\end{array}$ \\
\hline \multicolumn{2}{|c|}{ Autoimmune liver diseases } \\
\hline Primary biliary cholangitis & \multirow{3}{*}{$\begin{array}{c}\text { Refractive issues } 27.8 \%,{ }^{147} \text { Cataract } 12.5 \%,{ }^{147} \text { Dry eye and } \mathrm{KCS},{ }^{54,147} \text { Panuveitis, }{ }^{148} \text { Persistent subretinal, } \\
\text { Fluid due to central serous chorioretinopathy }{ }^{147,148}\end{array}$} \\
\hline Autoimmune hepatitis & \\
\hline Sclerosing cholangitis & \\
\hline \multicolumn{2}{|c|}{ Autoimmune neurological diseases } \\
\hline Multiple Sclerosis & $\begin{array}{c}\text { Ocular pain } 92 \%,{ }^{149} \text { Dyschromatopsia } 88 \%,{ }^{149} \text { ON } 25-75 \%,{ }^{150-152} \text { Intranuclear ophthalmoplegia } 30 \%,{ }^{153} \\
\text { Intermediate uveitis } 28.3 \%,{ }^{154} \text { Marcus Gunn pupil, }{ }^{155} \text { Retinal periphlebitis }{ }^{154}\end{array}$ \\
\hline Neuromyelitis Optica & $\begin{array}{c}\text { Decreased VA } 80 \%,{ }^{156,157} \text { Anterior ON } 40 \%,{ }^{156} \text { Retrobulbar ON } 36 \%,{ }^{156} \text { Oculomotor abnormalities, }{ }^{158} \\
\text { Intranuclear ophthalmoplegia }{ }^{158}\end{array}$ \\
\hline Myasthenia gravis & Ophthalmoparesis in eyelid elevators and extraocular muscles, ${ }^{159,160}$ Decreased VA, ${ }^{161}$ Dry eye. ${ }^{162}$ \\
\hline \multicolumn{2}{|c|}{ Other rheumatologic diseases } \\
\hline Fibromyalgia & Dry eye, ${ }^{49}$ Scleritis, ${ }^{163-165}$ Decreased retinal nerve fiber layer ${ }^{166}$ \\
\hline
\end{tabular}

are not common. $\mathrm{ON}$ is the most common diagnosis and can be present in $1 \%$ of SLE patients, ${ }^{45}$ which is similar to our results. Other uncommon diagnoses are PUK, scleritis, episcleritis, choroidopathy with serous detachments, orbit myositis, internuclear ophthalmoplegia, blepharospasm, and retinal necrosis. ${ }^{39}$ None of our patients presented these diagnoses.

FM is a disease characterized by chronic and generalized musculoskeletal pain. The etiology of this disease is still unknown. Although it is not classified as an autoimmune disease, it is considered a rheumatologic disease. $^{46,47}$ In our population FM was one of the most prevalent diseases, present in $22.71 \%$ of our patients. Ocular manifestations were present in $34.8 \%$, being DE the most frequent $(31.49 \%)$. In the same way, KCS was present in a significant proportion of these patients $(15.46 \%)$. There is a debate in the literature about the association of FM with DE. Aykut et $\mathrm{al}^{48}$ found that FM patients had increased corneal sensitivity and secondary eye discomfort related to dry eye disease, but there were no positive tests for dry eye disease. Contrarily, Vehof et $\mathrm{al}^{49}$ found that FM has an OR of 2.2 for DE. This does not help to make a clear statement in the face of this debate, so we think that larger case-control studies should be done to support some of these data. Additionally, some of our patients with FM presented uveitis, maculopathy, and $\mathrm{ON}$ as ocular manifestations. To the best of our knowledge, these ocular manifestations have not been previously reported. Although no statistically significant data relating to ocular manifestations and FM were found, novel related manifestations were described. These manifestations are an input to study a possible relationship between these variables in further research.

Ophthalmological symptoms and diseases related to other rheumatologic diseases that have been reported in the literature are shown in Table 5.

\section{Limitations}

It is well known that some drugs used for the treatment of rheumatological diseases can cause adverse effects or ocular toxicity, as in the case of chloroquine and corticosteroids. ${ }^{50}$ However, to control this confounding bias, this investigation included a temporality variable in its analysis, to ensure the relationship between ocular manifestations and diseases and the rheumatological disease.

One of the most important limitations was the lack of ophthalmologists in the rheumatology center. General practitioners and a rheumatologist were the ones that documented the main symptomatology and evident ophthalmological signs at the physical examination. 
When symptoms and/or signs were noted in the rheumatology consultation, patients usually assisted to an ophthalmological examination, and descriptions and findings were consigned in the patients' record. Nevertheless, this issue may generate an underestimation, resulting in a slight variation of the results.

Due to the medical records heterogeneity and the follow-up loss, diseases were newly classified according to the International Guidelines and the Tenth Edition of the International Classification of Diseases (ICD-10). The limitation lies in the difficulty of comparing some of the included diagnoses with other studies that used the ICD9 classification.

\section{Conclusion}

In our population, almost a third of patients reported ocular involvement. It is crucial to know its prevalence and the most common manifestations among the different rheumatologic diseases to offer early specialist referral and timely treatment. Dry eye corresponded to the main ocular manifestation, which requires strict monitoring to prevent severe complications. A multidisciplinary approach allows the monitoring of the disease and shared decisions on adequate therapy, enriching the knowledge of both parts and achieving the main objective, helping the patient.

\section{Abbreviations}

ADLT, Alejandra de-la-Torre; AS, Ankylosing spondylitis; ANCA, Antineutrophil Cytoplasmic Antibodies; APS, Antiphospholipid Syndrome; AD, Autoimmune Disease; Ads, Autoimmune Diseases; CCG, Carlos CifuentesGonzález; CLR, Carolina López-Rojas; CRAO, Central Retinal Artery Occlusion; CRVO, Central Retinal Vein Occlusion; DM, Dermatomyositis; DE, Dry Eye; EA, Enteropathic Arthritis; FMS, Fabien Mantilla-Sylvain; FM, Fibromyalgia; GCA, Giant Cell Arteritis; GD, Grave's disease; HT, Hashimoto Thyroiditis; HR, Hazard Ratio; HSP, Henoch-Schonlein purpura; HLA-B27, Human leukocyte antigen B27; IgA, Immunoglobulin A; IBD, Inflammatory Bowel Disease; JPTD, Juan Pablo Terreros-Dorado; JMO, Juliana Muñoz-Ortiz; JRG, Juliana Reyes-Guanes; JIA, Juvenile Idiopathic Arthritis; KCS, Keratoconjunctivitis Sicca; SL, Localized Scleroderma; LES, lupus erythematosus systemic; MCTD, Mixed Connective Tissue Disease; MS, Multiple Sclerosis; NMO, Neuromyelitis Optica; OR, Odds Ratio; ON, Optic Neuritis; PUK, Peripheral Ulcerative Keratitis; PUR, Pilar Uribe-Reina; PMR, Polymyalgia Rheumatica;
PM, Polymyositis; PsA, Psoriatic Arthritis; ReA, Reactive Arthritis; RS, Reiter Syndrome; AR, rheumatoid arthritis; RDMH, Rubén Darío Mantilla-Hernández; SS, Sjörgen Syndrome; SSc, Systemic Sclerosis; TA, Takayasu Arteritis; TAO, Thyroid Associated Ophthalmopathy; AV, Visual Acuity; WZ, William Zambrano.

\section{Data Sharing Statement}

The datasets used and/or analyzed during the current study are available by the corresponding author on reasonable request.

\section{Ethics Approval}

This study adheres to the ethical principles for human research established by the Helsinki Declaration, the Belmont Report, and Colombian Resolution 008430 of 1993. The confidentiality of the information has been preserved based on the Habeas data law (Organic Law 1581 of 2012). This investigation was presented to the research ethics committee of the Escuela Superior de Oftalmología del Instituto Barraquer de América, Bogotá, Colombia. However, as it is a retrospective study and according to the policies of the institution, it did require a registration process but did not require an ethics committee approval process.

\section{Acknowledgments}

We thank Funinderma for providing the clinical records.

\section{Author Contributions}

All authors made substantial contributions to conception and design, acquisition of data, or analysis and interpretation of data; took part in drafting the article or revising it critically for important intellectual content; agreed to submit to the current journal; gave final approval of the version to be published; and agree to be accountable for all aspects of the work.

\section{Disclosure}

The authors declare that they have no competing interests in this work.

\section{References}

1. Anaya J-M, Corena R, Castiblanco J, Rojas-Villarraga A, Shoenfeld Y. The kaleidoscope of autoimmunity: multiple autoimmune syndromes and familial autoimmunity. Expert Rev Clin Immunol. 2007;3 (4):623-635. doi:10.1586/1744666X.3.4.623

2. Anaya J-M. The autoimmune tautology. Arthritis Res Ther. 2010;12 (6):147. doi:10.1186/ar3175 
3. Selmi C, Brunetta E, Raimondo MG, Meroni PL. The $\mathrm{X}$ chromosome and the sex ratio of autoimmunity. Autoimmun Rev. 2012;11(6):A531-A537. doi:10.1016/j.autrev.2011.11.024

4. McCluskey P, Powell RJ. The eye in systemic inflammatory diseases. The Lancet. 2004;364(9451):2125-2133. doi:10.1016/ S0140-6736(04)17554-5

5. Agustí A, Faner R. Systemic Inflammation and Comorbidities in Chronic Obstructive Pulmonary Disease. Proc Am Thorac Soc. 2012;9(2):43-46. doi:10.1513/pats.201108-050MS

6. Generali E, Cantarini L, Selmi C. Ocular Involvement in Systemic Autoimmune Diseases. Clinic Rev Allerg Immunol. 2015;49(3):263-270. doi:10.1007/s12016-015-8518-3

7. Afshari NA, Afshari MA, Foster CS. Inflammatory conditions of the eye associated with rheumatic diseases. Curr Rheumatol Rep. 2001;3(5):453-458. doi:10.1007/s11926-996-0017-3

8. Troncoso LL, Biancardi AL. Ophthalmic manifestations in patients with inflammatory bowel disease: a review. World J Gastroenterol. 2017;23(32):5836-5848. doi:10.3748/wjg.v23.i32.5836

9. Foster CS, Forstot SL, Wilson LA. Mortality Rate in Rheumatoid Arthritis Patients Developing Necrotizing Scleritis or Peripheral Ulcerative Keratitis: effects of Systemic Immunosuppression. Ophthalmology. 1984;91(10):1253-1263. doi:10.1016/S01616420(84)34160-4

10. Riley GP, Harrall RL, Watson PG, Cawston TE, Hazleman BL. Collagenase (MMP-1) and TIMP-1 in destructive corneal disease associated with rheumatoid arthritis. Eye. 1995;9(6):703-718. doi:10.1038/eye. 1995.182

11. Kemeny-Beke A, Szodoray P. Ocular manifestations of rheumatic diseases. Int Ophthalmol. 2020;40(2):503-510. doi:10.1007/ s10792-019-01183-9

12. Varma R, Vajaranant TS, Burkemper B, et al. Visual Impairment and Blindness in Adults in the United States: demographic and Geographic Variations From 2015 to 2050. JAMA Ophthalmol. 2016;134(7):802-809. doi:10.1001/jamaophthalmol.2016.1284

13. Bourne RRA, Jonas JB, Bron AM, et al. Prevalence and causes of vision loss in high-income countries and in Eastern and Central Europe in 2015: magnitude, temporal trends and projections. $\mathrm{Br}$ $J$ Ophthalmol. 2018;102(5):575-585. doi:10.1136/bjophthalmol2017-311258

14. Akpek EK, Bunya VY, Saldanha IJ. Sjögren's Syndrome: more Than Just Dry Eye. Cornea. 2019;38(5):658-661. doi:10.1097/ ICO.0000000000001865

15. Kreutzmann A-K, Pannier S, Rojas-Perilla N, Schmid T, Templ M. The $R$ Package emdi for Estimating and Mapping Regionally Disaggregated Indicators. J Stat Soft. 2019;91(7). doi:10.18637/jss.v091.i07

16. Levitt AE, McManus KT, McClellan AL, Davis JL, Goldhardt R, Galor A. Ocular Inflammation in the Setting of Concomitant Systemic Autoimmune Conditions in an Older Male Population. Cornea. 2015;34(7):762-767. doi:10.1097/ICO.0000000000000437

17. Ciurtin C, Cojocaru VM, Aramă S, Stoica V. Epidemiology of ocular involvement in autoimmune diseases. Rom J Intern Med. 2008;46(3):243-247.

18. Ausayakhun S, Louthrenoo W, Aupapong S. Ocular diseases in patients with rheumatic diseases. J Med Assoc Thai. 2002;85 (8):855-862.

19. Pathanapitoon K, Tesavibul N, Choopong P, et al. Clinical manifestations of cytomegalovirus-associated posterior uveitis and panuveitis in patients without human immunodeficiency virus infection. JAMA Ophthalmol. 2013;131(5):638-645. doi:10.1001/jamaophthalmol.2013.2860

20. Adelowo OO, Akpabio AA, Oderinlo O, Okonkwo ON, Raphael J. Case Series and Literature Review of Ophthalmologic Disorders Seen in a Specialist Rheumatology Clinic in Lagos, Nigeria. Ocul Immunol Inflamm. 2019;27 (6):905-911. doi:10.1080/09273948.2019.1611873
21. Turk MA, Hayworth JL, Nevskaya T, Pope JE. Ocular manifestations in rheumatoid arthritis, connective tissue disease and vasculitis: a systematic review and meta-analysis. $J$ Rheumatol. 2020;48(1):25-34. doi:10.3899/jrheum.190768

22. Punjabi OS, Adyanthaya RS, Mhatre AD, Jehangir RP. Rheumatoid arthritis is a risk factor for dry eye in the Indian population. Ophthalmic Epidemiol. 2006;13(6):379-384. doi:10.1080/09286580600745969

23. Aboud S, Abd M. Ocular involvement and its manifestations in rheumatoid arthritis patients. Maedica. 2017;18:57-62. doi:10.4103/DJO.DJO_17_17

24. Vignesh APP, Srinivasan R. Ocular manifestations of rheumatoid arthritis and their correlation with anti-cyclic citrullinated peptide antibodies. Clin Ophthalmol. 2015;9:393-397. doi:10.2147/ OPTH.S77210

25. Stenstam T. On the Occurrence of Keratoconjunctivitis Sicca in Cases of Rheumatoid Arthritis. Acta Med Scand. 1947;127(12):130-148. doi:10.1111/j.0954-6820.1947.tb13134.x

26. Mody GM, Hill JC, Meyers OL. Keratoconjunctivitis sicca in rheumatoid arthritis. Clin Rheumatol. 1988;7(2):237-241. doi:10.1007/BF02204461

27. Cojocaru M, Cojocaru IM, Silosi I, Vrabie CD, Tanasescu R. Extra-articular Manifestations in Rheumatoid Arthritis. Maedica. 2010;5(4):286-291.

28. Harper SL, Foster CS. The ocular manifestations of rheumatoid disease. Int Ophthalmol Clin. 1998;38(1):1-19. doi:10.1097/ 00004397-199803810-00003

29. Bhadoria DP, Bhadoria P, Sundaram KR, Panda A, Malaviya AN. Ocular manifestations of rheumatoid arthritis. J Indian Med Assoc. 1989;87(6):134-135.

30. Lenoch F, Brémova A, Kankovax D, Streda A, Balik J. The Relation of SJögren's Syndrome to Rheumatoid Arthritis. Acta Rheumatol Scand. 1964;10(1-4):297-304. doi:10.3109/ rhe1.1964.10.issue-1-4.33

31. Reddy SC, Gupta SD, Jain IS, Deodhar SD. Ocular manifestations of rheumatoid arthritis. Indian J Ophthalmol. 1977;25 (3):20-26.

32. Baer AN, Walitt B. Update on Sjögren Syndrome and Other Causes of Sicca in Older Adults. Rheum Dis Clin North Am. 2018;44(3):419-436. doi:10.1016/j.rdc.2018.03.002

33. Devauchelle-Pensec V, Mariette X, Jousse-Joulin S, et al. Treatment of primary Sjögren syndrome with rituximab: a randomized trial. Ann Intern Med. 2014;160(4):233-242. doi:10.7326/M13-1085

34. Brito-Zerón P, Acar-Denizli N, Zeher M, et al. Influence of geolocation and ethnicity on the phenotypic expression of primary Sjögren's syndrome at diagnosis in 8310 patients: a crosssectional study from the Big Data Sjögren Project Consortium. Ann Rheum Dis. 2017;76(6):1042-1050. doi:10.1136/annrheumdis-2016-209952

35. Parke AL, Buchanan WW. Sjögren's syndrome: history, clinical and pathological features. Inflammopharmacology. 1998;6 (4):271-287. doi:10.1007/s10787-998-0012-6

36. Safonova TN, Iudina IV. Characteristics of keratoconjunctivitis sicca in Sjögren's disease and syndrome. Ter Arkh. 1988;60 (4):30-31.

37. Friedlaender MH. Ocular manifestations of Sjögren's syndrome: keratoconjunctivitis sicca. Rheum Dis Clin North Am. 1992;18 (3):591-608. doi:10.1016/S0889-857X(21)00318-5

38. Dammacco R. Systemic lupus erythematosus and ocular involvement: an overview. Clin Exp Med. 2018;18(2):135-149. doi:10.1007/s10238-017-0479-9

39. Papagiannuli E, Rhodes B, Wallace GR, Gordon C, Murray PI, Denniston AK. Systemic lupus erythematosus: an update for ophthalmologists. Surv Ophthalmol. 2016;61(1):65-82. doi:10.1016/j.survophthal.2015.06.003 
40. Silpa-archa S, Lee JJ, Foster CS. Ocular manifestations in systemic lupus erythematosus. $\mathrm{Br} J$ Ophthalmol. 2016;100 (1):135-141. doi:10.1136/bjophthalmol-2015-306629

41. Palejwala NV, Walia HS, Yeh S. Ocular manifestations of systemic lupus erythematosus: a review of the literature. Autoimmune Dis. 2012;2012:290898. doi:10.1155/2012/290898

42. Read RW. Clinical mini-review: systemic lupus erythematosus and the eye. Ocul Immunol Inflamm. 2004;12(2):87-99. doi:10.1080/09273940490895308

43. Jensen JL, Bergem HO, Gilboe IM, Husby G, Axéll T. Oral and ocular sicca symptoms and findings are prevalent in systemic lupus erythematosus. J Oral Pathol Med. 1999;28(7):317-322. doi:10.1111/j.1600-0714.1999.tb02047.x

44. Steinberg AD, Talal N. The coexistence of Sjögren's syndrome and systemic lupus erythematosus. Ann Intern Med. 1971;74 (1):55-61. doi:10.7326/0003-4819-74-1-55

45. Stafford-Brady FJ, Urowitz MB, Gladman DD, Easterbrook M. Lupus retinopathy. Patterns, associations, and prognosis. Arthritis Rheum. 1988;31(9):1105-1110. doi:10.1002/art.17803 10904

46. Clauw DJ. Fibromyalgia: a clinical review. JAMA. 2014;311 (15):1547-1555. doi:10.1001/jama.2014.3266

47. Goldenberg DL. Fibromyalgia syndrome. An emerging but controversial condition. JAMA. 1987;257(20):2782-2787. doi:10.1001/jama.1987.03390200122026

48. Aykut V, Elbay A, Çigdem Uçar I, et al. Corneal sensitivity and subjective complaints of ocular pain in patients with fibromyalgia. Eye. 2018;32(4):763-767. doi:10.1038/eye.2017.275

49. Vehof J, Snieder H, Jansonius N, Hammond CJ. Prevalence and risk factors of dry eye in 79,866 participants of the population-based Lifelines cohort study in the Netherlands. Ocul Surf. 2020. doi:10.1016/j.jtos.2020.04.005

50. Peponis V, Kyttaris V, Chalkiadakis S, Bonovas S, Sitaras N. Review: ocular side effects of anti-rheumatic medications: what a rheumatologist should know. Lupus. 2010;19(6):675-682. doi:10.1177/0961203309360539

51. Gilboe I-M, Kvien TK, Uhlig T, Husby G. Sicca symptoms and secondary Sjögren's syndrome in systemic lupus erythematosus: comparison with rheumatoid arthritis and correlation with disease variables. Ann Rheum Dis. 2001;60(12):1103-1109. doi:10.1136 ard.60.12.1103

52. Brun JG, Madland TM, Jonsson R. A prospective study of sicca symptoms in patients with rheumatoid arthritis. Arthritis Care Res. 2003;49(2):187-192. doi:10.1002/art.10999

53. Fousekis FS, Katsanos A, Katsanos KH, Christodoulou DK. Ocular manifestations in celiac disease: an overview. Int Ophthalmol. 2020;40(4):1049-1054. doi:10.1007/s10792019-01254-x

54. Alarcón-Segovia D, Díaz-Jouanen E, Fishbein E. Features of Sjögren's Syndrome in Primary Biliary Cirrhosis. Ann Intern Med. 1973;79(1):31-36. doi:10.7326/0003-4819-79-1-31

55. Gomes B, Santhiago MR, Magalhães P, Kara-Junior N. Ocular findings in patients with systemic sclerosis. Clinics. 2011;66 (3):379-385. doi:10.1590/S1807-59322011000300003

56. Szucs G, Szekanecz Z, Aszalos Z, et al. A Wide Spectrum of Ocular Manifestations Signify Patients with Systemic Sclerosis. Ocul Immunol Inflamm. 2019:1-9. doi:10.1080/09273948.20 19.1657467

57. Tailor R, Gupta A, Herrick A, Kwartz J. Ocular Manifestations of Scleroderma. Surv Ophthalmol. 2009;54(2):292-304. doi:10.1016/j.survophthal.2008.12.007

58. Horan EC. Ophthalmic manifestations of progressive systemic sclerosis. Br J Ophthalmol. 1969;53(6):388-392. doi:10.1136/ bjo.53.6.388

59. West RH, Barnett AJ. Ocular involvement in scleroderma. $\mathrm{Br}$ J Ophthalmol. 1979;63(12):845-847. doi:10.1136/bjo.63.12.845
60. Waszczykowska A, Goś R, Waszczykowska E, DziankowskaBartkowiak B, Jurowski P. Clinical researchPrevalence of ocular manifestations in systemic sclerosis patients. Arch Med Sci. 2013;9(6):1107-1113. doi:10.5114/aoms.2013.39217

61. Zulian F, Vallongo C, Woo P, et al. Localized scleroderma in childhood is not just a skin disease. Arthritis Rheum. 2005;52 (9):2873-2881. doi:10.1002/art.21264

62. Lythgoe H, Almeida B, Bennett J, et al. Multi-centre national audit of juvenile localised scleroderma: describing current UK practice in disease assessment and management. Pediatric Rheumatol. 2018;16(1):80. doi:10.1186/s12969-018-0295-0

63. Allanore Y, Parc C, Monnet D, Brézin AP, Kahan A. Increased prevalence of ocular glaucomatous abnormalities in systemic sclerosis. Ann Rheum Dis. 2004;63(10):1276-1278. doi:10.1136/ ard.2003.013540

64. Kim J-H, Joo Y-B, Kim J, Min J-K. A case of hypertrophic cranial pachymeningitis presenting with scleritis in a patient with undifferentiated connective tissue disease. $J$ Korean Med Sci. 2010;25(6):966-969. doi:10.3346/jkms.2010.25.6.966

65. Protti A, Erminio C, Piccolo I, Spreafico C, Colombo F, Ghezzi A. An unusual case with relapsing neuromyelitis optica associated with undifferentiated connective tissue disease. Neurol Sci. 2004;25 (Suppl 4):S383-385. doi:10.1007/s10072-004-0348-7

66. Colon LE, Enzenauer RJ. Corneal thinning as the sole manifestation of active undifferentiated connective tissue disease. South Med J. 1990;83(1):48-50. doi:10.1097/00007611-19900100000015

67. Chylack LT, Bienfang DC, Bellows AR, Stillman JS. Ocular Manifestations of Juvenile Rheumatoid Arthritis. Am J Ophthalmol. 1975;79(6):1026-1033. doi:10.1016/00029394(75)90689-3

68. Heiligenhaus A, Niewerth M, Ganser G, Heinz C, Minden K. German Uveitis in Childhood Study Group. Prevalence and complications of uveitis in juvenile idiopathic arthritis in a population-based nation-wide study in Germany: suggested modification of the current screening guidelines. Rheumatology. 2007;46(6):1015-1019. doi:10.1093/rheumatology/kem053

69. Latif EMAE, Derini GFE. Study of the different ocular manifestations in children with juvenile idiopathic arthritis. J Egyptian Ophthalmol Soc. 2017;110(2):31. doi:10.4103/ejos.ejos_15_17

70. EL-Shereef RR, Lofty G, Shawkat A. Ocular Manifestation of Juvenile Idiopathic Arthritis and its Relation to Disease Activity. $J$ Arthritis. 2014;3(3):137. doi:10.4172/2167-7921.1000137

71. Alarcon-Segovia D. Symptomatic Sjögren's syndrome in mixed connective tissue disease. J Rheumatol. 1976;3(2):191-195.

72. Domngang Noche C, Kagmeni G, Dohvoma V, Bella AL, Ebana Mvogo C, Singwe-Ngandeu M. Ophthalmic Manifestations in Chronic Inflammatory Rheumatic Diseases at a Referral Hospital of Yaounde, Cameroon. Ocul Immunol Inflamm. 2018;26(2):259-264. doi:10.1080/09273948.2016.1212078

73. Graf WD, Milstein JM, Sherry DD. Stroke and mixed connective tissue disease. J Child Neurol. 1993;8(3):256-259. doi:10.1177/ 088307389300800309

74. Mimura T, Usui T, Amano S, et al. Retinal vasculitis and vitreous hemorrhage associated with mixed connective tissue disease: retinal vasculitis in MCTD. Int Ophthalmol. 2005;26(45):159-161. doi:10.1007/s10792-006-9015-8

75. Nadeau SE. Neurologic manifestations of connective tissue disease. Neurol Clin. 2002;20(1):151-178. doi:10.1016/s07338619(03)00057-4

76. Hunter DG. Ocular manifestations of sarcoidosis. In: Albert DM, Jakobiec FA, editors. Principles and Practice of Ophthalmology. 1994:443-450.

77. Ohara K, Okubo A, Sasaki H, Kamata K. Intraocular manifestations of systemic sarcoidosis. Jpn J Ophthalmol. 1992;36 (4):452-457. 
78. Atmaca LS, Atmaca-Sönmez P, Idil A, Kumbasar OO, Celik G. Ocular involvement in sarcoidosis. Ocul Immunol Inflamm. 2009;17(2):91-94. doi:10.1080/09273940802596526

79. Pasadhika S, Rosenbaum JT. Ocular Sarcoidosis. Clin Chest Med. 2015;36(4):669-683. doi:10.1016/j.ccm.2015.08.009

80. Matsou A, Tsaousis KT. Management of chronic ocular sarcoidosis: challenges and solutions. Clin Ophthalmol. 2018;12:519-532. doi:10.2147/OPTH.S128949

81. Saari R, Lahti R, Saari KM, et al. Frequency of rheumatic diseases in patients with acute anterior uveitis. Scand J Rheumatol. 1982;11(2):121-123. doi:10.3109/03009748209098175

82. Stolwijk C. Prevalence of extra-articular manifestations in patients with ankylosing spondylitis: a systematic review and meta-analysis. Ann Rheum Dis. 2015;74(1):65-73. doi:10.1136/ annrheumdis-2013-203582

83. Zeboulon N, Dougados M, Gossec L. Prevalence and characteristics of uveitis in the spondyloarthropathies: a systematic literature review. Ann Rheum Dis. 2008;67(7):955-959. doi:10.1136/ ard.2007.075754

84. Marsovszky L, Németh J, Resch MD, et al. Corneal Langerhans cell and dry eye examinations in ankylosing spondylitis. Innate Immun. 2014;20(5):471-477. doi:10.1177/1753425913498912

85. Abbouda A, Abicca I, Fabiani C, et al. Psoriasis and Psoriatic Arthritis-Related Uveitis: different Ophthalmological Manifestations and Ocular Inflammation Features. Semin Ophthalmol. 2017;32 (6):715-720. doi:10.3109/08820538.2016.1170161

86. Lima FBF, Abalem MF, Ruiz DG, et al. Prevalence of eye disease in Brazilian patients with psoriatic arthritis. Clinics. 2012;67 (3):249-253. doi:10.6061/clinics/2012(03)08

87. Turk M, Hayworth J, Nevskaya T, Pope J. Sat0352 the Frequency of Ocular Manifestations in Patients with Adult Vs. Childhood Spondyloarthritis. Ann Rheum Dis. 2019;78(Supp12):1256. doi:10.1136/annrheumdis-2019-eular.2434

88. Malaviya AN, Agrawal N, Patil NS. Clinical characteristics of peripheral spondyloarthritis without psoriasis, inflammatory enteropathy or preceding infection, from a single rheumatology clinic in northern India. Clin Rheumatol. 2017;36(11):2613-2618. doi:10.1007/s10067-017-3720-8

89. Mintz R, Feller ER, Bahr RL, Shah SA. Ocular Manifestations of Inflammatory Bowel Disease. Inflamm Bowel Dis. 2004;10 (2):135-139. doi:10.1097/00054725-200403000-00012

90. Felekis T, Katsanos K, Kitsanou M, et al. Spectrum and Frequency of Ophthalmologic Manifestations in Patients with Inflammatory Bowel Disease: a Prospective Single-Center Study. Inflamm Bowel Dis. 2009;15(1):29-34. doi:10.1002/ ibd.20584

91. Faez S, Lobo A-M, Unizony SH, Stone JH, Papaliodis GN, Sobrin L. Ocular inflammatory disease in patients with polymyalgia rheumatica: a case series and review of the literature. Clin Rheumatol. 2016;35(1):251-258. doi:10.1007/s10067-014-2558-6

92. Molina N, Gonzalez-Gonzalez LA, Doctor PP, Tauber J, Foster CS. Clinical Characteristics of a Large Cohort of Patients with Scleritis and Episcleritis. Ophthalmology. 2012;119 (1):43-50. doi:10.1016/j.ophtha.2011.07.013

93. Arai T, Tanaka R, Kaburaki T. Ocular Inflammation Associated with Polymyalgia Rheumatica without Concomitant Giant-Cell Arteritis: a Report of Three Cases. Ocul Immunol Inflamm. 2018;26(5):779-782. doi:10.1080/09273948.2017.1278776

94. Sobolewska B, Zierhut M. Dermatomyositis. In: Zierhut M, Pavesio C, Ohno S, Orefice F, Rao NA, editors. Intraocular Inflammation. Berlin, Heidelberg: Springer; 2016:815-819. doi:10.1007/978-3-540-75387-2 69

95. Kahaly GJ, Diana T, Glang J, Kanitz M, Pitz S, König J. Thyroid Stimulating Antibodies Are Highly Prevalent in Hashimoto's Thyroiditis and Associated Orbitopathy. J Clin Endocrinol Metab. 2016;101(5):1998-2004. doi:10.1210/jc.2016-1220
96. Kan E, Kılıçkan E, Ecemiş G, Beyazyildiz E, Çolak R. Presence of Dry Eye in Patients with Hashimoto's Thyroiditis. J Ophthalmol. 2014;2014:754923. doi:10.1155/2014/754923

97. Altin Ekin M, Karadeniz Ugurlu S, Egrilmez ED, Oruk GG. Ocular Surface Changes in Hashimoto's Thyroiditis Without Thyroid Ophthalmopathy. Eye Contact Lens. 2020. doi:10.1097/ ICL.0000000000000686

98. Gharib S, Moazezi Z, Bayani MA. Prevalence and severity of ocular involvement in Graves' disease according to sex and age: a clinical study from Babol, Iran. Caspian J Intern Med. 2018;9 (2):178-183. doi:10.22088/cjim.9.2.178

99. Tanda ML, Piantanida E, Liparulo L, et al. Prevalence and natural history of Graves' orbitopathy in a large series of patients with newly diagnosed graves' hyperthyroidism seen at a single center. J Clin Endocrinol Metab. 2013;98(4):1443-1449. doi:10.1210/jc.2012-3873

100. Sayin N, Kara N, Pekel G. Ocular complications of diabetes mellitus. World J Diabetes. 2015;6(1):92-108. doi:10.4239/wjd. v6.i1.92

101. Ljubimov AV. Diabetic complications in the cornea. Vision Res. 2017;139:138-152. doi:10.1016/j.visres.2017.03.002

102. Cousen P, Cackett P, Bennett H, Swa K, Dhillon B. Tear production and corneal sensitivity in diabetes. J Diabetes Complicat. 2007;21(6):371-373. doi:10.1016/j.jdiacomp.2006.05.008

103. Asherson RA, Merry P, Acheson JF, Harris EN, Hughes GR. Antiphospholipid antibodies: a risk factor for occlusive ocular vascular disease in systemic lupus erythematosus and the "primary" antiphospholipid syndrome. Ann Rheum Dis. 1989;48 (5):358-361. doi:10.1136/ard.48.5.358

104. Demirci FY, Küçükkaya R, Akarçay K, et al. Ocular involvement in primary antiphospholipid syndrome. Ocular involvement in primary APS. Int Ophthalmol. 1998;22(6):323-329. doi:10.1023/a:1006305705080

105. Cervera R, Boffa M-C, Khamashta MA, Hughes GRV. The Euro-Phospholipid project: epidemiology of the antiphospholipid syndrome in Europe. Lupus. 2009;18(10):889-893. doi:10.1177/ 0961203309106832

106. Castañón C, Amigo M-C, Bañales JL, Nava A, Reyes PA. Ocular Vaso-occlusive Disease in Primary Antiphospholipid Syndrome. Ophthalmology. 1995;102(2):256-262. doi:10.1016/S01616420(95)31028-7

107. Yehudai D, Shoenfeld Y, Toubi E. Looking into the eyes of patients with antiphospholipid syndrome. Clin Rev Allergy Immunol. 2007;32(2):192-197. doi:10.1007/s12016-007-0002-2

108. Pulido JS, Ward LM, Fishman GA, Goodwin JA, Froelich CJ, Sanghvi JP. Antiphospholipid antibodies associated with retinal vascular disease. Retina. 1987;7(4):215-218. doi:10.1097/ 00006982-198707040-00002

109. Levine SR, Brey RL. Neurological aspects of antiphospholipid antibody syndrome. Lupus. 1996;5(5):347-353. doi:10.1177/ 096120339600500503

110. Miserocchi E, Baltatzis S, Foster CS. Ocular features associated with anticardiolipin antibodies: a descriptive study. $\mathrm{Am}$ $J \quad$ Ophthalmol. 2001;131(4):451-456. doi:10.1016/S00029394(00)00884-9

111. Takkar B, Khokhar S, Kumar U, Venkatesh P. Necrotising scleritis, keratitis and uveitis in primary antiphospholipid syndrome. BMJ Case Rep. 2018;2018. doi:10.1136/bcr-2017-220647

112. Majji AB, Bhatia K, Mathai A. Spontaneous bilateral peripapillary, subhyaloid and vitreous hemorrhage with severe anemia secondary to idiopathic thrombocytopenic purpura. Indian $J$ Ophthalmol. 2010;58(3):234-236. doi:10.4103/0301-4738.62651

113. Shah PA, Yang SS, Fung WE. Idiopathic Thrombocytopenic Purpura With Massive Subretinal Hemorrhage. Arch Ophthalmol. 2005;123(11):1612-1613. doi:10.1001/ archopht.123.11.1612 
114. Awh CC, Modjtahedi BS, Eliott D. Purtscher's retinopathy as the presenting manifestation of immune thrombocytopenic purpura. Am J Ophthalmol Case Rep. 2017;6:77-80. doi:10.1016/j. ajoc.2017.03.001

115. Chiu S, Lim B, Chan N Progressive Retinopathy In Adult Onset Idiopathic Thrombocytopenia Purpura. 2005. Available from: https://www.aaopt.org/detail/knowledge-base-article/progressiveretinopathy-adult-onset-idiopathic-thrombocytopenia-purpura. Accessed July 6, 2020.

116. Karagiannis D, Gregor Z. Valsalva retinopathy associated with idiopathic thrombocytopenic purpura and positive antiphospholipid antibodies. Eye. 2006;20(12):1447-1449. doi:10.1038/sj. eye. 6702313

117. Honig SE, Srinivasan A, Shields CL. Suprachoroidal Hemorrhage Simulating Melanoma in Idiopathic Thrombocytopenic Purpura. Ocul Oncol Pathol. 2019;5(3):162-166. doi:10.1159/000490390

118. Foulds WS, Chisholm IA, Stewart JB, Wilson TM. The Optic Neuropathy of Pernicious Anemia. Arch Ophthalmol. 1969;82 (4):427-432. doi:10.1001/archopht.1969.00990020429001

119. Hamilton HE, Ellis PP, Sheets RF. Visual Impairment due to Optic Neuropathy in Pernicious Anemia: report of a Case and Review of the Literature. Blood. 1959;14(4):378-385. doi:10.1182/blood.V14.4.378.378

120. Troncoso J, Mancall EL, Schatz NJ. Visual Evoked Responses in Pernicious Anemia. Arch Neurol. 1979;36(3):168-169. doi:10.1001/archneur.1979.00500390086010

121. Lerman S, Feldmahn AL. Centrocecal Scotomata as the Presenting Sign in Pernicious Anemia. Arch Ophthalmol. 1961;65(3):381-385. doi:10.1001/archopht.1961.01840020383011

122. Ellis PP, Hamilton H. Retrobulbar Neuritis in Pernicious Anemia*. Am J Ophthalmol. 1959;48(1):95-97. doi:10.1016/ 0002-9394(59)90291-0

123. Chew FLM, Tajunisah I. Retinal phlebitis associated with autoimmune hemolytic anemia. Ocul Immunol Inflamm. 2009;17 (6):394-395. doi:10.3109/09273940903260204

124. Oner A, Ozkiris A, Dogan H, Erkilic K, Karakukcu M. BILATERAL MACULAR HEMORRHAGE ASSOCIATED WITH AUTOIMMUNE HEMOLYTIC ANEMIA. RETINA. 2005;25(8):1089-1090. doi:10.1097/00006982-200512000-00018

125. Sharma A, Dhooria A, Aggarwal A, Rathi M, Chandran V. Connective Tissue Disorder-Associated Vasculitis. Curr Rheumatol Rep. 2016;18(6):31. doi:10.1007/s11926-016-0584-x

126. Schmidt D, Lagrèze W, Vaith P. Ophthalmoscopic findings in 3 patients with panarteritis nodosa and review of the literature. Klin Monbl Augenheilkd. 2001;218(1):44-50. doi:10.1055/s-2001-11260

127. Akova YA, Jabbur NS, Foster CS. Ocular Presentation of Polyarteritis Nodosa: clinical Course and Management with Steroid and Cytotoxic Therapy. Ophthalmology. 1993;100 (12):1775-1781. doi:10.1016/S0161-6420(93)31405-3

128. Miyanaga M, Takase H, Ohno-Matsui K. Anti-Neutrophil Cytoplasmic Antibody-Associated Ocular Manifestations in Japan: a Review of 18 Patients. Ocul Immunol Inflamm. 2020;1-6. doi:10.1080/09273948.2020.1712432

129. Razzak A, Kassimi A, Mchachi A, et al. Eil et Maladie de Behçet: profil épidémiologique, clinique, thérapeutique et évolutif. Pan Afr Med J. 2019;33. doi:10.11604/ pamj.2019.33.116.17111

130. Paovic J, Paovic P, Sredovic V. Behcet's Disease: systemic and Ocular Manifestations. Biomed Res Int. 2013;2013. doi:10.1155/ 2013/247345

131. Cullen JF, Coleiro JA. Ophthalmic complications of giant cell arteritis. Surv Ophthalmol. 1976;20(4):247-260. doi:10.1016/ 0039-6257(76)90224-1

132. McCluskey $P$, Powell RJ. The eye in systemic inflammatory diseases. The Lancet. 2004;364(9451):2125-2133. doi:10.1016/ S0140-6736(04)17554-5
133. Sook Chun Y, Park S-J, Kipark I, Chung H, Lee J. THE CLINICAL AND OCULAR MANIFESTATIONS OF TAKAYASU ARTERITIS. RETINA. 2001;21(2):132-140. doi:10.1097/00006982-200104000-00006

134. Muqit MMK, Gallagher MJ, Gavin M, Roberts F, Jardine AG. Henoch-Schonlein purpura with keratitis and granulomatous anterior uveitis. $\mathrm{Br} J$ Ophthalmol. 2005;89(9):1221-1222. doi:10.1136/bjo.2004.064519

135. Chuah J, Meaney T. Anterior ischaemic optic neuropathy secondary to Henoch-Schönlein Purpura. Eye. 2005;19(9):1028. doi:10.1038/sj.eye.6701764

136. Nicholson L, Mathews D. Bilateral cystoid macular oedema and cotton wool spots associated with Henoch-Schonlein purpura. Case Reports. 2013;2013:bcr2013200159. doi:10.1136/bcr-2013200159

137. Lorentz WB, Weaver RG. Eye Involvement in Anaphylactoid Purpura. Am J Dis Child. 1980;134(5):524-525. doi:10.1001/ archpedi.1980.02130170074027

138. Erbagci I, Erbagci Z, Gungor K, Bekir N. Ocular anterior segment pathologies and tear film changes in patients with psoriasis vulgaris. Acta Med Okayama. 2014.

139. Chandran NS, Greaves M, Gao F, Lim L, Cheng BCL. Psoriasis and the eye: prevalence of eye disease in Singaporean Asian patients with psoriasis. J Dermatol. 2007;34(12):805-810. doi:10.1111/j.1346-8138.2007.00390.x

140. Reed WB, Becker SW. Psoriasis and Arthritis. AMA Arch Derm. 1960;81(4):577-585. doi:10.1001/archderm.1960.03730040081015

141. Cruz NFSD, Brandão LS, Cruz SFSD, Cruz SASD, Pires CAA, Carneiro FRO. Ocular manifestations of psoriasis. Arq Bras Oftalmol. 2018;81(3):219-225. doi:10.5935/0004-2749.20180044

142. Baskan EB, Baykara M, Ercan İ, Tunali S, Yucel A. Vitiligo and ocular findings: a study on possible associations. J Eur Acad Dermatol Venereol. 2006;20(7):829-833. doi:10.1111/j.14683083.2006.01655.x

143. Biswas G, Barbhuiya JN, Biswas MC, Islam MN, Dutta S. Clinical pattern of ocular manifestations in vitiligo. $J$ Indian Med Assoc. 2003;101(8):478-480.

144. Dogan AS, Atacan D, Durmazlar SPK, Acar M, Gurdal C. Evaluation of dry eye findings in patients with vitiligo. Pak J Med Sci. 2015;31(3):587-591. doi:10.12669/pjms.313.6926

145. España A, Iranzo P, Herrero-González J, Mascaro JM, Suárez R. Ocular involvement in pemphigus vulgaris - a retrospective study of a large Spanish cohort. JDDG. 2017;15(4):396-403. doi:10.1111/ddg. 13221

146. Foster CS. Cicatricial pemphigoid. Trans Am Ophthalmol Soc. 1986;84:527-663.

147. Citirik M, Berker N, Kacar S, Kekilli M. Ocular Findings in Patients with Autoimmune Liver Disease. Ocul Immunol Inflamm. 2012;20 (6):438-442. doi:10.3109/09273948.2012.719994

148. Kamal A, Bhan A, Murray PI. Uveitis with autoimmune hepatic disorders. Ocul Immunol Inflamm. 2001;9(4):267-272. doi:10.1076/ocii.9.4.267.3960

149. The clinical profile of optic neuritis. Experience of the Optic Neuritis Treatment Trial. Optic Neuritis Study Group. Arch Ophthalmol. 1991;109(12):1673-1678. doi:10.1001/archopht.1991.01080120 057025

150. Atkins EJ, Biousse V, Newman NJ. Optic neuritis. Semin Neurol. 2007;27(3):211-220. doi:10.1055/s-2007-979683

151. Miller D, Barkhof F, Montalban X, Thompson A, Filippi M. Clinically isolated syndromes suggestive of multiple sclerosis, part I: natural history, pathogenesis, diagnosis, and prognosis. Lancet Neurol. 2005;4(5):281-288. doi:10.1016/S1474-4422(05) 70071-5

152. Graves J, Balcer LJ. Eye disorders in patients with multiple sclerosis: natural history and management. Clin Ophthalmol. 2010;4:1409-1422. doi:10.2147/OPTH.S6383 
153. Müri RM, Meienberg O. The Clinical Spectrum of Internuclear Ophthalmoplegia in Multiple Sclerosis. Arch Neurol. 1985;42 (9):851-855. doi:10.1001/archneur.1985.04060080029011

154. Kardes E, Sezgin Akcay BI, Bozkurt K, Unlu C, Erdogan G, Akcali G. Clinical characteristics of intermediate uveitis in adult Turkish patients. Int $J$ Ophthalmol. 2015;8(4):759-763. doi:10.3980/j.issn.2222-3959.2015.04.21

155. Nilsson P, Larsson E-M, Maly-Sundgren P, Perfekt R, SandbergWollheim M. Predicting the outcome of optic neuritis: evaluation of risk factors after 30 years of follow-up. $J$ Neurol. 2005;252 (4):396-402. doi:10.1007/s00415-005-0655-9

156. Merabtene L, Vignal Clermont C, Deschamps R. Neuropathie optique dans le syndrome des anticorps anti MOG positif. $J$ Français d'Ophtalmologie. 2019;42(10):1100-1110. doi:10.1016/j.jfo.2019.06.006

157. Levin MH, Bennett JL, Verkman AS. OPTIC NEURITIS IN NEUROMYELITIS OPTICA. Prog Retin Eye Res. 2013;36:159-171. doi:10.1016/j.preteyeres.2013.03.001

158. Sun H, Cui S, Gao F, et al. Eye movement abnormalities in AQP4-IgG positive neuromyelitis optica spectrum disorder. J Neurol Sci. 2018;384:91-95. doi:10.1016/j.jns.2017.11.033

159. Gilhus NE, Tzartos S, Evoli A, Palace J, Burns TM, Verschuuren JJGM. Myasthenia gravis. Nat Rev Dis Primers. 2019;5(1):30. doi:10.1038/s41572-019-0079-y
160. Gilhus NE, Verschuuren JJ. Myasthenia gravis: subgroup classification and therapeutic strategies. Lancet Neurol. 2015;14 (10):1023-1036. doi:10.1016/S1474-4422(15)00145-3

161. Roh HS, Lee SY, Yoon JS. Comparison of Clinical Manifestations between Patients with Ocular Myasthenia Gravis and Generalized Myasthenia Gravis. Korean J Ophthalmol. 2011;25(1):1-7. doi:10.3341/kjo.2011.25.1.1

162. Wang T-J, Wang I-J, Hu -C-C, Lin H-C. Comorbidities of dry eye disease: a nationwide population-based study. Acta Ophthalmol. 2012;90(7):663-668. doi:10.1111/j.1755-3768.2010.01993.x

163. Zdebik N, Zdebik A, Bogusławska J, Przeździecka-Dołyk J, Turno-Kręcicka A. Fibromyalgia Syndrome and Eye - a review. Surv Ophthalmol. 2020. doi:10.1016/j.survophthal.2020.05.006

164. Fan N, Florakis G. Scleritis Associated with the Fibromyalgia Syndrome. Cornea. 1996;15(6):637-638. doi:10.1097/00003226199611000-00017

165. Chow K, Emara B. Necrotizing scleritis and fibromyalgia: a potential link? Can $J$ Ophthalmol. 2008;43(1):117-118. doi:10.3129/i07-207

166. Pilar Bambo M, Garcia-Martin E, Gutierrez-Ruiz F, et al. Study of perfusion changes in the optic disc of patients with fibromyalgia syndrome using new colorimetric analysis software. J Français d'Ophtalmologie. 2015;38(7):580-587. doi:10.1016/ j.jfo.2015.01.010
Clinical Ophthalmology

\section{Publish your work in this journal}

Clinical Ophthalmology is an international, peer-reviewed journal covering all subspecialties within ophthalmology. Key topics include: Optometry; Visual science; Pharmacology and drug therapy in eye diseases; Basic Sciences; Primary and Secondary eye care; Patient Safety and Quality of Care Improvements. This journal is indexed on PubMed

\section{Dovepress}

Central and CAS, and is the official journal of The Society of Clinical Ophthalmology (SCO). The manuscript management system is completely online and includes a very quick and fair peer-review system, which is all easy to use. Visit http://www.dovepress.com/ testimonials.php to read real quotes from published authors. 